Angular momentum transport during the formation and early evolution of stars - EES2012

P.Hennebelle and C. Charbonnel

EAS Publications Series, Vol. ??, 2018

\title{
MRI-DRIVEN ANGULAR MOMENTUM TRANSPORT IN PROTOPLANETARY DISKS
}

\author{
Sébastien Fromang ${ }^{1}$
}

\begin{abstract}
Angular momentum transport in accretion disk has been the focus of intense research in theoretical astrophysics for many decades. In the past twenty years, MHD turbulence driven by the magnetorotational instability has emerged as an efficient mechanism to achieve that goal. Yet, many questions and uncertainties remain, among which the saturation level of the turbulence. The consequences of the magnetorotational instability for planet formation models are still being investigated. This lecture, given in September 2012 at the school "Role and mechanisms of angular momentum transport in the formation and early evolution of stars" in Aussois (France), aims at introducing the historical developments, current status and outstanding questions related to the magnetorotational instability that are currently at the forefront of academic research.
\end{abstract}

\section{Introduction}

"In this paper and a companion work, we show that a broad class of astrophysical accretion disk is dynamically unstable to axisymmetric disturbances in the presence of a weak magnetic field." (Balbus and Hawlev, 1991)

The above sentence is opening the soon to be seminal paper of Balbus and Hawley (1991). It brought to the scene a magnetohydrodynamical instability that destabilizes magnetized accretion disks. Although known since the 60's Velikhov, 1959; Chandrasekhar, 1961), its importance in the field of astrophysics had not been appreciated. In the few years that followed, it was rapidly realized that the magnetorotational instability (or, in short, MRI), as it was to be called, has profound consequences for the dynamics of accretion disks. It is now believed to be the main physical effect responsible for the fall of matter onto the object (young star, white

\footnotetext{
${ }^{1}$ Laboratoire AIM, CEA/DSM-CNRS-Université Paris Diderot, IRFU/Service d'Astrophysique, CEA-Saclay F-91191 Gif-sur-Yvette, France
} 
dwarf, neutron star or black hole) that sits at the center of accretion disks, thus solving a long lasting mystery of modern astrophysics. Its consequences cannot be ignored when studying such important issues as planet formation or black hole physics. In just two decades, the paper by Balbus and Hawley (1991) changed the field of accretion disk astrophysics.

This lecture is aimed at $\mathrm{PhD}$ students, postdocs and researchers that are nonspecialist of that field. Its purpose is to establish as simply as possible the basic physical mechanisms associated with the MRI come into play and to highlight the outstanding questions we currently struggle with. Even if some aspects of the lecture have broad implications to all categories of accretion disks, it tends to be focused on the particular case of protoplanetary (PP) disks, i.e. accretion disks rotating around newly born stars. Since PP disks are the nurseries of planetary systems such as our own, research devoted to understanding their properties has been intense ever since the discovery of the MRI. As we shall see, the conditions that prevail in PP disks result in peculiarities that greatly complicate their dynamics. Many outstanding questions remain for future generations to solve.

Before moving on, let me emphasize that this lecture is not a review about the MRI. It is meant to be an introduction to the topic at the most basic level and it is strongly biased toward PP disks. For a more complete overview of the MRI, especially for those issues that are specific to other classes of disks than just PP disks, the interested reader will benefit from the published reviews by Balbus and Hawley (1998); Balbus and Hawley (2000); Balbus (2003). The subject of PP disks itself is very rich and many aspects of PP disks not related to the MRI are left aside in this lecture. Excellent reviews on PP disks have recently been published that provide a comprehensive overview of their properties. The interested reader is referred for example to Dullemond and Monnier (2010) or Armitage (2011) for completeness.

Angular momentum transport in accretion disks Before moving to the physics of the MRI and its consequences, it is first important to understand the stakes of the problem. What are we after? The question we are facing is quite simple: matter in accretion disks falls onto the central object, and we want to understand why. The answer is not as simple as it may sound, though! To first order, matter in accretion disks describes circular orbits around the central object (we will note its mass $M$ in the following). This is much like the earth orbiting around the sun. The force balance is between the central gravitational attraction and the centrifugal force. In Newtonian dynamics, that balance results in the orbital velocity $v_{\phi}$ of each element being Keplerian:

$$
v_{\phi}=R \Omega=\sqrt{\frac{G M}{R}} .
$$

In the above equation, $G$ is the gravitational constant and $R$ is the distance to the central object. The parameter $\Omega$ is called the angular velocity and is an important parameter of this course. The important point of that formula, though, 
is that matter possesses a finite amount of angular momentum per unit mass, $L=R v_{\phi}=R^{2} \Omega$, that it must lose in order to be accreted onto the central object. This requires dissipative effects that are able to extract angular momentum from each element. Otherwise, angular momentum is conserved and matter, like the earth, will stay for ever on its circular Keplerian orbit. The problem of mass accretion, thus, translates into the central theme of this book: angular momentum transport.

How can angular momentum be extracted from matter in accretion disks? The answer to that question is easy, isn't it? As we just saw, accretion disks are in Keplerian rotation, which means the angular velocity is varying with radius. Or, in other words, the flow is sheared: elements sitting on neighboring orbits rotate at different rates. This is fortunate as we know from fluid mechanics courses that the fluid molecular viscosity $\nu$ exerts a force between two differentially rotating rings. The inner ring is slowed down by the outer (and more slowly rotating) ring. Stated in terms of angular momentum conservation, this means that angular momentum flows from the inner ring to the outer ring: the inner ring's angular momentum decreases and the ring itself moves in as a result. In other words it accretes toward the central object. The problem of accretion disk is solved! Unfortunately, things are not that simple and it turns out that this naive picture badly fails. Consider indeed the timescale associated with this diffusive process. The typical time to transport matter over the distance $R$ is given by the viscous timescale

$$
t_{\nu}=\frac{R^{2}}{\nu} .
$$

An order of magnitude estimate of the viscosity can be obtained as follows. It is of the order of the thermal velocity of the molecules $v_{t h}$ times their mean free path $\lambda: \nu \sim v_{t h} \lambda . v_{t h}$ can be estimated, for molecules of mass $m$ in a medium at temperature $\mathrm{T}$, through the relation

$$
\frac{1}{2} m_{H_{2}} v_{t h}^{2} \sim \frac{3}{2} k_{B} T,
$$

where $k_{B}$ is the Boltzmann constant. For hydrogen molecules (of mass $m_{H_{2}}=$ $3.4 \times 10^{-27} \mathrm{~kg}$ ) at a temperature of about $100 \mathrm{~K}$ typical of PP disks, we find $v_{t h} \sim 1 \mathrm{~km} \cdot \mathrm{s}^{-1}$. The molecules mean free path can be approximated by

$$
\lambda \sim \frac{1}{n_{H_{2}} \sigma} .
$$

Here, $\sigma \sim \pi a^{2} \sim 3 \times 10^{-16} \mathrm{~cm}^{-2}$ is the geometrical cross section between molecules (we have taken $a=1$ Angström as a typical molecular size). $n_{H_{2}}$ is the number of molecules per unit volume. If we call $\Sigma$ the disk surface density and $H$ its thickness, then $n_{H_{2}} m_{H_{2}} \sim \Sigma / H$. In PP disks, typical values of the surface density at 1 Astronomical Unit (AU) from the central star are of order $10^{3} \mathrm{~g} . \mathrm{cm}^{-2}$, while $H / R \sim 0.05$. Thus we find, at $1 \mathrm{AU}, n_{H_{2}} \sim 4 \times 10^{14} \mathrm{~cm}^{-3}$. Putting things 
together, we obtain $\lambda \sim 0.1-1 \mathrm{~m}\left[1\right.$, from which $\nu \sim 10^{3} \mathrm{~m}^{2} . \mathrm{s}^{-1}$ follows. We are now in a position to evaluate the viscous timescale of PP disks. At $1 \mathrm{AU}$, we obtain $t_{\nu} \sim 10^{18} \mathrm{~s}$. This is about $10^{11} \mathrm{yr}$, i.e. much longer than the age of the universe! Surely, a more efficient mechanism must exist to account for the observation that PP disks lifetime amounts to a few million years.

Our intuition based on laboratory experiment as well as everyday life is that turbulence could well be such a mechanism. Indeed, turbulence is known to be efficient at transporting things around. In the same way the turbulent air in a room can rapidly transport molecules of perfume from one side of that room to the other, turbulence in accretion disks could well transport angular momentum from their inner regions to their outer parts. This is why people quickly made the ansatz that accretion disks are turbulent. This hypothesis is further supported by the realization that the Reynolds number $R e$ of the flow is gigantic in accretion disks. Using the above estimates for the various physical parameters of the system, $R e$ can indeed be estimated to be

$$
R e=\frac{v_{\phi} H}{\nu} \sim 10^{11} \gg 1
$$

Laboratory experiments tell us that fluid flows become turbulent at high Reynolds number, even when they are linearly stable as is the case of Keplerian flow (a result known as the Rayleigh criterion that we will recover in section 2.2). This is known as nonlinear instability. Is it also the case of accretion disks? Well, despite decades of intense research and vivid controversy, the nonlinear stability of accretion disks is still debated and there is no accepted argument, be it theoretical, numerical or experimental, that accretion disks are hydrodynamically turbulent. We shall return to that issue in more details in section 4.1. For the purpose of this introduction, it is sufficient to note that the source of turbulence in accretion disks remained elusive for many years.

Models of accretion disks, however, were made possible by the introduction in the 70's of the so-called $\alpha$ disks model in two seminal papers written by Shakura and Sunyaev (1973) and Lynden-Bell and Pringle (1974). Such models are in fact large scale models of the turbulence, different versions of which are commonly used in other field of physics. The idea is to replace the molecular viscosity discussed above by an anomalous (i.e. boosted) viscosity that is supposed to mimic the effect of turbulence. How unsatisfactory as it may seem, such an approach generally give helpful insights, qualitative and even sometimes quantitative agreements with real flows. As we saw above, viscosity has the dimensions of a velocity times a length. Using the sound speed $c_{s}$ as a characteristic velocity and the disk thickness as a characteristic length, Shakura and Sunyaev (1973) and Lynden-Bell and Pringle (1974) introduced the following scaling for the viscosity

$$
\nu_{t}=\alpha c_{s} H \text {, }
$$

\footnotetext{
${ }^{1}$ We note in passing that the mean free path of the gas is much smaller than the dimension of the system. We will thus adopt a fluid description to described its dynamics at the scales of interest (typically of order a fraction of $H$ ) for this lecture.
} 


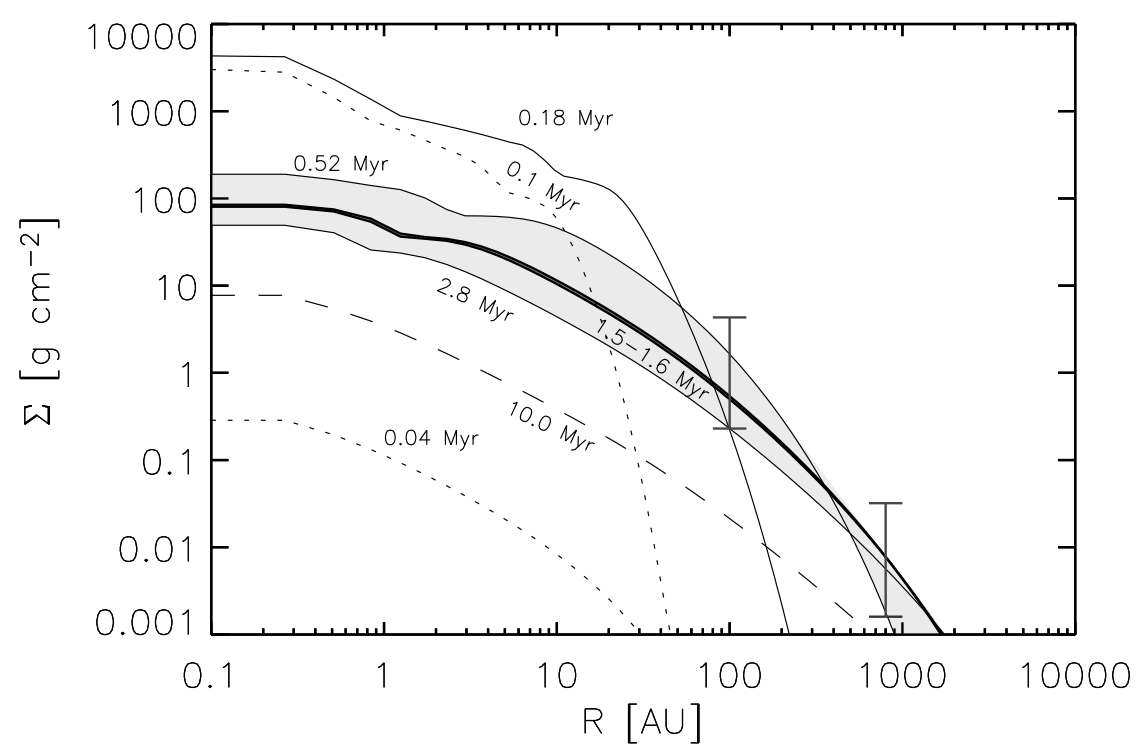

Fig. 1. Radial profile of the surface density obtained when solving the diffusion equation given in a typical $\alpha$ disk model. The different lines correspond to different times during the disk evolution, the vertical errors bars show the contraints provided by observations of DM Tau and the grey shaded area shows the ensemble of models that fit those constraints. Figure extracted from Hueso and Guillot (2005), to which the reader is referred for further details on the model parameters.

where $\alpha$ is a dimensionless quantity, supposedly constant, after which the model is named. The subscript $t$ serves to differentiate turbulent and molecular viscosity. Using that scaling for the viscosity, it is possible to build detailed and time dependent models of accretion disks. For example, the disk surface density satisfies the following diffusion equation as a result of mass and angular momentum conservation

$$
\frac{\partial \Sigma}{\partial t}=\frac{3}{R} \frac{\partial}{\partial R}\left(R^{1 / 2} \frac{\partial}{\partial R}\left(\nu R^{1 / 2} \Sigma\right)\right) .
$$

Such an equation can be used to calculate the time evolution of $\Sigma$. It is not the purpose of this lecture, however, to provide an extensive review of $\alpha$-disk models. The interested reader is referred to the classical textbook by Frank et al. (2002) that exposes the details of such models. In the introduction of this lecture, we shall content ourselves with an order of magnitude estimate of $\alpha$.

What range of possible values can we expect? There are basically two constraints. The first comes from the fact that turbulent eddies should have a typical scale $l_{t}$ 
smaller than $H$ and that motions should be subsonic (i.e. the velocity fluctuations $\delta v_{t} \leq c_{s} \sqrt{2}$. As seen above, molecular viscosity can be estimated as the product between the typical velocity of the molecules and the typical distance they travel between collisions. By analogy, turbulent viscosity can be estimated to be of order $l_{t} \delta v_{t}$. The constraints on $l_{t}$ and $\delta v_{t}$ suggest an upper bound of order $c_{s} H$ for the turbulent viscosity. In other words, we expect $\alpha \leq 1$. A second constraint on $\alpha$ comes from the typical evolution timescale $t_{\mathrm{PP}}$ of PP disks. Observations suggest that $t_{\mathrm{PP}} \sim 10^{6-7}$ years (Armitage, 2011). Using the same reasoning as for molecular viscosity, we can write

$$
t_{\mathrm{PP}} \sim \frac{R_{d}^{2}}{\nu_{t}}
$$

where $R_{d} \sim 100 \mathrm{AU}$ is now the disk outer radius. Using $\nu_{t}=\alpha c_{s} H$ and $c_{s}=H \Omega$, we find 3

$$
\alpha \sim \frac{1}{2 \pi}\left(\frac{R}{H}\right)^{2}\left(\frac{T_{\text {orb }}\left(R=R_{d}\right)}{t_{P P}}\right) \sim 10^{-2} \text { to } 10^{-1}
$$

This argument can be carried a bit further as done for example by Hueso and Guillot (2005). These authors solved the diffusion equation for the disk surface density given by Eq. (1.7), adding a source term on the right hand side of the equation. Such a term is meant to model the infall of material from the envelope out of which the protostar formed. The disk midplane temperature (required to evaluate the turbulent viscosity) is calculated by including the effect of turbulent heating, irradiation from the central star and radiative cooling from the disk surface. Using reasonable initial conditions, they calculated the time evolution of the radial profile of the surface density and compared their results with observational constraints for two objects. The typical results they obtained are illustrated on figure 1 To account for millimeter observations of DM Tau, Hueso and Guillot (2005) concluded that $\alpha$ should be in the range $10^{-3}$ to $10^{-1}$. Similar constraints were drawn for the star GM Aur. These values are in broad agreement with the simple orders of magnitude described above. The paper by Hueso and Guillot (2005) is interesting because it illustrates the possible use of $\alpha$-disk models in PP disks. It also highlights that even detailed comparison with the observations only results in very loose constraints on the transport of angular momentum in PP disks that are no better than order of magnitude estimates. This is not only due to the limits of the observations themselves (limited angular resolution, uncertainties in the age of the objects) but also to the simplicity of the $\alpha$-model, which assumes spatial as well as temporal constancy of $\alpha$ and reduces turbulence as a simple viscosity.

\footnotetext{
${ }^{2}$ Indeed, any supersonic motion would quickly be damped because of shocks. Likewise, turbulent structures at scales larger than $\mathrm{H}$ would produce supersonic differential motions because of the background shear

${ }^{3}$ In a locally isothermal disk (i.e. the gas temperature is independent of the distance $Z$ to the disk midplane), hydrostatic equilibrium in the vertical direction writes $\partial P / \partial Z=-\rho G M /\left(R^{2}+\right.$ $\left.Z^{2}\right)^{3 / 2}$. When $Z \ll R$ and using $P=\rho c_{s}^{2}$, one finds by integration that $\rho \propto \exp \left(-Z^{2} / 2 H^{2}\right)$, with $H=c_{s} / \Omega$ and $\Omega=\sqrt{G M / R^{3}}$ is the gas midplane angular velocity.
} 
The lesson not to forget from this discussion is that $\alpha$ disk models are rough large scale models that can at best offer an order of magnitude estimate of accretion disks properties. Using them beyond there domain of validity is dangerous business. They can serve as useful guides, especially when it comes to comparing theoretical expectations with observations, but understanding the underlying mechanisms that power the turbulence is mandatory if we want to address all aspects of accretion disk dynamics and angular momentum transport. This lecture is designed to explore the properties of the most popular of these mechanisms, namely the magnetorotational instability.

Outline of the lecture The rest of the course is divided in three parts. The first part focuses on the linear instability. A simple derivation is provided that is used to extract the key properties of the MRI and to describe its physical mechanism. The particular problems that arise in the case of PP disks are emphasized. The second part is a tour of twenty years of numerical simulations of the MRI. The techniques, results and limits of those simulations are discussed. Finally, the last part highlights a set key aspects of planet formation theories that interface with the MRI. Outstanding issues are highlighted and discussed along the way.

\section{The Magnetorotational Instability}

The goal of the present section is essentially to present a simple derivation of the MRI. It will serve to establish the basic properties of the instability. Despite the simplifications adopted in this section (geometry of the field, locality of the analysis, over-simplified treatment of the energetics), most of its conclusions are general.

\subsection{Governing equations}

We begin by deriving a simplified form of the dynamical equations. Our starting point is the equations of ideal MHD (see chapter 1). Several approximations are made that significantly simplify the derivation of the MRI dispersion relation.

First, we assume the equation of state is isothermal: $P=\rho c_{0}^{2}$, where $c_{0}$ is the constant sound speed of the gas. This removes the energy equation from the problem. We next define a Cartesian coordinate system centered at a radial distance $R=R_{0}$ from the central mass and rotating around the central star with angular velocity $\Omega_{0}$. The $\mathrm{x}$-axis points in the radial direction, the $\mathrm{y}$-axis is directed along the direction of the flow rotation while the $\mathrm{z}$-direction is perpendicular to the equatorial plane of the disk (see figure 2). The associated unit vectors are noted $\left(\boldsymbol{e}_{\boldsymbol{x}}, \boldsymbol{e}_{\boldsymbol{y}}, \boldsymbol{e}_{\boldsymbol{z}}\right)$. We next consider a small volume within the disk. For all points within that domain, we thus have in particular $x \ll R_{0}$. In addition to pressure and the Coriolis force, each fluid element is subject to the gravitational and centrifugal accelerations. In the former, we neglect the vertical component of the force because of the box small size (in practice, it means we ignore the vertical density stratification of the disk). The combined effect of gravitational and centrifugal acceleration 


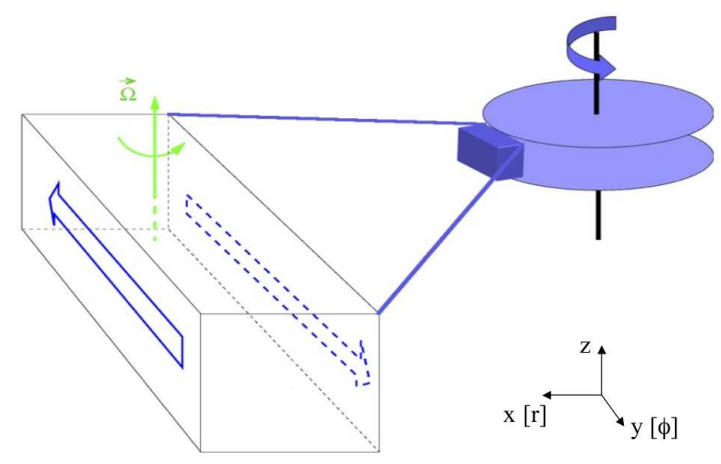

Fig. 2. Cartoon illustrating the local approach and coordinate system adopted for the derivation of the MRI dispersion relation (section 2) and for shearing boxes numerical simulations (section 3). Figure courtesy G. Lesur.

can be expressed as that of an equivalent tidal potential $\phi_{\text {eff }}$. If we assume that the gas angular velocity follows a power law, $\Omega \propto R^{-q}$, the tidal potential satisfies the relation 4

$$
\nabla \phi_{\mathrm{eff}}=-R \Omega^{2} \boldsymbol{e}_{\boldsymbol{x}}+R \Omega_{0}^{2} \boldsymbol{e}_{\boldsymbol{x}}=2 q \Omega_{0}^{2} x \boldsymbol{e}_{\boldsymbol{x}}
$$

where we have used $R=R_{0}+x$ and a Taylor expansion that results from the smallness of the domain. Straightforward integration yields:

$$
\phi_{\mathrm{eff}}=q \Omega_{0}^{2} x^{2} .
$$

We are now in a position to write the equations governing the fluid evolution. These equations are simply the MHD equations discussed in the first chapter of this book, augmented with the effective potential $\phi_{\text {eff }}$ and the Coriolis force. Neglecting all dissipative terms (i.e. we work in the ideal MHD limit), we obtain:

$$
\begin{aligned}
\frac{\partial \rho}{\partial t}+\nabla \cdot(\rho \boldsymbol{v}) & =0, \\
\rho\left[\frac{\partial \boldsymbol{v}}{\partial t}+(\boldsymbol{v} \cdot \boldsymbol{\nabla}) \boldsymbol{v}\right]+2 \rho \boldsymbol{\Omega}_{\mathbf{0}} \times \boldsymbol{v} & =-\boldsymbol{\nabla} P+\frac{1}{4 \pi}(\boldsymbol{\nabla} \times \boldsymbol{B}) \times \boldsymbol{B}+2 q \rho \Omega_{0}^{2} x \boldsymbol{e}_{\boldsymbol{x}}(2.4) \\
\frac{\partial \boldsymbol{B}}{\partial t} & =\boldsymbol{\nabla} \times(\boldsymbol{v} \times \boldsymbol{B}) .
\end{aligned}
$$

\footnotetext{
${ }^{4}$ For Keplerian flow, of course, $q=3 / 2$. We don't specialize to that case in the following, though, as we want to examine the stability of the flow as a function of its angular momentum radial profile.
} 
As usual, we have adopted the following notations: $\rho$ stands for the gas density, $\boldsymbol{v}$ for the velocity, $\boldsymbol{B}$ for the magnetic field, $P$ for the pressure, while $\boldsymbol{\Omega}_{\mathbf{0}}=\Omega_{0} \boldsymbol{e}_{\boldsymbol{z}}$.

\subsection{Dispersion relation: a simple derivation}

We now consider the simplest possible case where the system is threaded by a uniform vertical magnetic field of strength $\boldsymbol{B}=B_{0} \boldsymbol{e}_{\boldsymbol{z}}$. In equilibrium, the gas density is uniform: $\rho=\rho_{0}$.

In such a situation, the equilibrium state is a balance between the Coriolis force and centrifugal acceleration. It leads to $v_{y}=-q \Omega x$. There is a linear shear. The stability of that equilibrium is studied by considering small perturbations. For simplicity, we consider here perturbations that only depend on $z$ and $t$. All variables will thus be the sum of the equilibrium and time independent part (denoted with a "0" subscript and the perturbation (denoted with a prime exponent). For example, the density writes:

$$
\rho=\rho_{0}+\rho^{\prime}(z, t) .
$$

The linearized equations on the perturbed density and vertical velocity decouple from the other equations and write:

$$
\begin{aligned}
\frac{\partial \rho^{\prime}}{\partial t}+\frac{\partial}{\partial z}\left(\rho_{0} v_{z}^{\prime}\right) & =0 \\
\rho_{0} \frac{\partial v_{z}^{\prime}}{\partial t}+c_{0}^{2} \frac{\partial \rho^{\prime}}{\partial z} & =0
\end{aligned}
$$

These two equations correspond to standard sound waves propagating in the vertical direction. They are not affected by the background rotation. The $z$ component of the induction equation simply gives:

$$
\frac{\partial B_{z}^{\prime}}{\partial t}=0
$$

Together with the condition $\boldsymbol{\nabla} \cdot \boldsymbol{B}=0$, which translates to the vertical derivative of $B_{z}^{\prime}$ vanishing, this means that $B_{z}^{\prime}$ is a constant that can be incorporated into $B_{0}$.

The remaining four equations ( $x$ and $y$ components of the momentum and induction equations) give:

$$
\begin{aligned}
\rho_{0} \frac{\partial v_{x}^{\prime}}{\partial t} & =-c_{0}^{2} \frac{\partial \rho^{\prime}}{\partial z} \\
\rho_{0}\left[\frac{\partial v_{y}^{\prime}}{\partial t}-q \Omega v_{x}^{\prime}\right] & =B_{0} \frac{\partial B_{y}^{\prime}}{\partial z}-2 \rho_{0} \Omega v_{x}^{\prime} \\
\frac{\partial B_{x}^{\prime}}{\partial t} & =B_{0} \frac{\partial v_{x}^{\prime}}{\partial z} \\
\frac{\partial B_{y}^{\prime}}{\partial t} & =-q \Omega B_{x}^{\prime}+B_{0} \frac{\partial v_{y}^{\prime}}{\partial z} .
\end{aligned}
$$


The set of equations derived above is homogeneous in $z$ and $t$. This means we can take the Fourier transform by writing all variables as:

$$
X^{\prime}=\overline{X^{\prime}} \exp (i(\omega t-k z)) \text {. }
$$

Upon adopting this definition, the four equations describing horizontal motions and magnetic field fluctuations then lead to the following algebraic expressions:

$$
\begin{aligned}
i \omega \rho_{0} v_{x}^{\prime} & =-i k B_{0} B_{x}^{\prime} \\
i \omega \rho_{0} v_{y}^{\prime} & =q \rho_{0} \Omega v_{x}^{\prime}-i k B_{0} B_{y}^{\prime}-2 \rho_{0} \Omega v_{x}^{\prime} \\
i \omega B_{x}^{\prime} & =-i k B_{0} v_{x}^{\prime} \\
i \omega B_{y}^{\prime} & =-q \Omega B_{x}^{\prime}-i k B_{0} v_{y}^{\prime}
\end{aligned}
$$

where the overlines in the above expressions have been dropped for clarity. Combining these equations leads after some algebra to the following quartic equation that constitutes the dispersion relation of the problem:

$$
\omega^{4}-\omega^{2}\left[2 k^{2} v_{A}^{2}+\kappa^{2}\right]+k^{2} v_{A}^{2}\left[k^{2} v_{A}^{2}-2 q \Omega^{2}\right]=0,
$$

where $\kappa^{2}=2(2-q) \Omega^{2}$ is the square of the epicyclic frequency and $v_{A}=B_{0} / \sqrt{\rho_{0}}$ the Alfven velocity.

In the absence of a magnetic field, $v_{A}=0$ and the dispersion relation reduces to:

$$
\omega^{2}-\kappa^{2}=0
$$

Thus, the stability condition in hydrodynamic disks is $\kappa^{2} \geq 0$, or equivalently $q \leq 2$. This is the Rayleigh criterion: accretion disks with angular momentum increasing outward are linearly stable.

The presence of a magnetic field dramatically modifies that classical result. The sum $s$ of the two roots $\omega_{ \pm}^{2}$ of Eq. (2.19) is given by $2 s=2 k^{2} v_{A}^{2}+\kappa^{2}$. It is always positive, which means that their product determines the stability condition:

$$
k^{2} v_{A}^{2}-2 q \Omega^{2} \geq 0 .
$$

The stability condition is thus $q \leq 0$. In the opposite case, $q \geq 0$, there is always a critical value $k_{c}^{2}=2 q \Omega^{2} / v_{A}^{2}$ of the wavenumber such that $k^{2} v_{A}^{2}-2 q \Omega^{2}$ is negative for all $k<k_{c}$. Because of the definition of $q$, the system is unstable whenever the angular velocity decreases outward, a condition that is readily satisfied in accretion disks. A remarkable feature of the MRI, first noted by Chandrasekhar (1961), is that the condition for the flow to be destabilized by the magnetic field does not depend on the strength of the field itself. This is because the Lorentz force depends on the variations of the magnetic field along each field line: the small scale oscillations of a small B-field results in the same Lorentz force as the large scale oscillation of a large field (Balbus and Hawley, 1991). 


\subsection{MRI properties}

The fastest growing mode Using equation (2.19), the most unstable mode is characterized by a growth rate $\sigma_{\max }$ and a wavenumber $k_{\max }$ that satisfy the following relations:

$$
\begin{aligned}
\sigma_{\max } & =\frac{q \Omega}{2} \\
k_{\max }^{2} v_{A}^{2} & =\frac{q}{4}(4-q) \Omega^{2} .
\end{aligned}
$$

For accretion disks in Keplerian rotation, $q=3 / 2$ which results in $\sigma_{\max }=0.75 \Omega$ and $k_{\max } v_{A} \sim \Omega$. As noticed early on (Balbus and Hawley, 1991, 1998), this is an enormous growth rate as it results in amplification factors in energy per dynamical time larger than $10^{4}$ (Balbus and Hawley, 1998). In fact, Balbus and Hawley (1992) conjectured that the MRI growth rate is the fastest growth rate for instabilities resulting from differential rotation. This is one of the reason the MRI quickly became important in dynamical studies of accretion disks.

The structure of the most unstable mode of the MRI consists in horizontal layers of alternating radial velocities and radial magnetic fields that grow in amplitude. The action of shear means that azimuthal velocities and magnetic fields are produced. Vertical velocity and density perturbations both vanish (which means that the most unstable mode of the MRI is incompressible). These growing "fingers" of perturbed magnetic fields and velocities are now known as channel modes.

A weak field instability The instability criterion derived above states that all modes of wavelength larger than a critical scale (given by the wavenumber $k_{c}$ ) are unstable. Of course, these unstable modes cannot be larger in scale than the typical vertical size of the system. A necessary condition for the MRI to operate is thus that the smallest of those modes fit within the disk:

$$
k_{c} \geq \frac{2 \pi}{H}
$$

This equation can be written as a condition on the plasma parameter $\beta$, the ratio between thermal and magnetic pressure:

$$
\beta \geq \frac{4 \pi^{2}}{q}
$$

Since the parameters entering the right hand side of this relation are all of order unity, this is a requirement that the magnetic field strength should be smaller than a given threshold for which magnetic and thermal energy are roughly in equipartition. This is why the MRI is often qualified as being a weak field instability.

Physical interpretation The physical mechanism of the instability is illustrated in figure 3. Consider two fluid elements sitting on a particular magnetic 


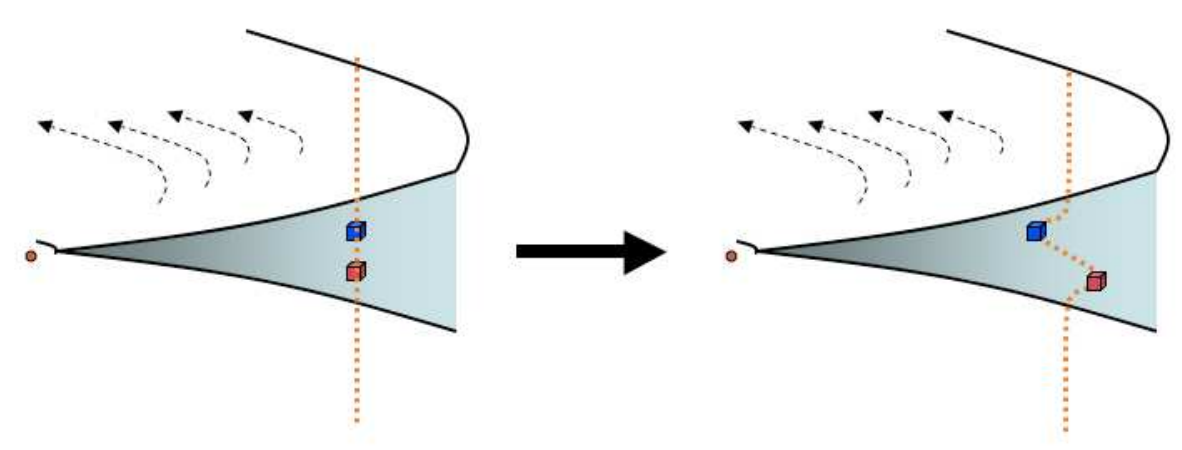

Fig. 3. Cartoon illustrating physical mechanism of the MRI (the black dashed line shows the radially decreasing angular velocity of the gas). Consider two fluid elements at the same cylindrical radius threading a weak vertical field line (shown with the red dotted line), then displaced as shown in the figure. To maintain their respective angular momenta, the (upper) inward moving mass rotates more rapidly and the (lower) outward moving mass less rapidly. This creates an azimuthal tension force in the field line that transfers angular momentum from the inwardly displaced mass to the outwardly displaced mass. Since the inward mass loses angular momentum it drifts further inward; the outward mass, acquiring angular momentum, moves further outward. This increases the field line tension and the process runs away.

field line that is purely vertical when unperturbed. When that field line is perturbed, these two fluid elements are displaced and follow the field line perturbation (this is because, in the ideal MHD limit, fluid particles and magnetic fields are tied together). They move to different radial location. In order to conserve their angular momenta, they start to rotate at different angular velocity and begin drifting apart. This drift stretches the magnetic field line that link the two fluid particles and results in a magnetic force (the increased tension of that field lines) on both fluid elements. That force slows (accelerates) the inner (outer) fluid element which moves further inward (outward) in a runaway process. In the meantime, angular momentum has been exchanged between them.

A robust instability The situation considered above was deliberately chosen to be oversimplified in order to highlight the basic properties of the instability and the physical mechanism at play. Focus has been placed on the simplest perturbations to highlight the physical property of the instability. Axisymmetry has been assumed and we have considered perturbations that do not depend on $x$. In fact, there exists other modes with a finite $k_{x}$ (Balbus and Hawley, 1991) as well as non-axisymmetric modes (Hawley and Balbus, 1992). They have smaller growth rates than the channel modes discussed above. In addition, the MRI grows in conditions that are more general than the simple setup considered above. the 
MRI grows in the presence of a radial magnetic field (Balbus and Hawley, 1991) or a pure azimuthal magnetic field (Hawley and Balbus, 1992). In this latter case, the perturbations are required to be non axisymmetric, which renders the analysis quite complicated because of the background shear, and the growth is only transient for finite vertical wavenumbers. Normal modes also exist when the vertical density stratification of the disk is accounted for (Gammie and Balbus, 1994). To state things simply, the MRI grows as long as a weak magnetic field is present in a sheared rotating flow where angular velocity decreases outward.

\subsection{The case of protoplanetary disks}

As described above, the MRI is a powerful linear instability that displays enormous growth rates in the ideal MHD limit. However, an implicit assumption of the above analysis is that there is good coupling between the gas and the magnetic field. We have assumed that the gas is a perfect conductor. However, in cold and dense environments such as in PP disks, this is not the case. We will see below that simple estimates of the electron fraction in PP disks confirm that this can significantly alter the MRI properties describing above. Before doing so, we focus on the requirement for the MRI to operate when the effect of dissipation coefficients is taken into account.

The effect of diffusion coefficients The MRI dispersion relation was derived above in the limit of dissipationless MHD. As mentioned already, such an assumption is not realistic because of the large plasma diffusivity. This is why various authors have explored the influence of a finite resistivity and viscosity (Lesur and Longaretti, 2007; Pessah and Chan, 2008). The results of such studies is not surprising: dissipation tends to reduce the growth rate of the instability. The detailed modifications on both the growth rate and the eigenmodes can be analyzed in details but turns out to be complicated. However, it is possible to use simple scaling arguments that illustrate their importance for PP disks. Here we follow the discussion of Fleming et al. (2000) who focused on the case where dissipation is dominated by a large ohmic resistivity $\eta$. In such a situation, the induction equation given by Eq. (2.5) is modified and writes

$$
\frac{\partial \boldsymbol{B}}{\partial t}=\boldsymbol{\nabla} \times(\boldsymbol{v} \times \boldsymbol{B}-\eta \boldsymbol{\nabla} \times \boldsymbol{B}) .
$$

The additional term in the induction equation is responsible for diffusing the magnetic field. As a result, MRI modes with a wavenumber $k$ are expected to be affected when their growth rate is of order the diffusion rate $\eta k^{2}$ associated with that diffusivity. For the most unstable MRI mode, the growth rate is of order $\Omega$ and is thus affected by Ohmic resistivity when:

$$
\eta k^{2} \sim \Omega .
$$


In addition, its wavenumber $k$ roughly satisfies $k v_{A} \sim \Omega$. Combining the two expression, we can expect the MRI to be stabilized when

$$
\Lambda=\frac{v_{A}^{2}}{\eta \Omega} \leq 1
$$

The dimensionless parameter $\Lambda$ is called the Elsasser number. Of course, small scale modes (large $k$ ) are affected first by the resistivity while larger and larger resistivity is required to stabilize the large scale modes (small $k$ ) and the simple argument above is only meant to give an order of magnitude estimate. Nevertheless, the simple criterion that the Elsasser number needs to be larger than unity for the MRI to operate is confirmed by more detailed analysis.

Now since the resistivity is a decreasing function of electron abundance, the above criterion also shows that a small electron fraction will tend to stabilize the flow. Let us try to be more quantitative. What is the typical electron fraction that is required at $1 \mathrm{AU}$ in a typical PP disk? To answer that question, Blaes and Balbus (1994) used standard expressions for the conductivity, such as given by Spitzer (1962) for typical astrophysical plasmas. They provide a closed form for the resistivity in a disk consisting of ions and neutrals:

$$
\eta=\frac{230 T^{1 / 2}}{x_{e}} \mathrm{~cm}^{2} \cdot \mathrm{s}^{-1} .
$$

In the following we consider typical values for the disk parameters at $1 \mathrm{AU}$, i.e. $c_{s}=$ $1 \mathrm{~km} . \mathrm{s}^{-1}, \Omega=2 \times 10^{-7} \mathrm{~s}^{-1}$ and a temperature of a few hundreds Kelvin. We can expect the magnetic field to reach at most equipartition (an expectation confirmed by numerical simulations), so that we take $v_{A} \sim c_{s}$. Combining Eq. (2.29) with the requirement that $\Lambda$ should be larger than unity, we see that the boundary between stable and unstable flows lies at $x_{e} \sim 10^{-13}$. This is an extremely small number, but we shall see in the following that there are indeed regions of the disks where the electron fraction is much smaller.

PP disks are cold The simplest way to produce electrons in gases is through thermal ionization produced by collisions between particles. Temperature in PP disks range from a few thousands Kelvins in their inner regions to a few tens of Kelvins. The number of electrons produced by thermal ionization in such circumstances can be worked out using the Saha equation. For atoms with a single level of ionization, it writes (Spitzer, 1962)

$$
\frac{n_{e}^{2}}{n_{i}}=\frac{2}{\Lambda_{B}^{3}} \frac{g_{1}}{g_{0}} \exp \left(-\frac{\epsilon}{k_{B} T}\right) \text { where } \Lambda_{B}=\sqrt{\frac{h^{2}}{2 \pi m_{e} k_{B} T}}
$$

is the thermal de Broglie wavelength, which amounts to $\sim 2.4 \times 10^{-7} \mathrm{~cm}$ when $T=10^{3} \mathrm{~K}$. The other terms are the density of electrons $n_{e}$ and ions $n_{i}$, their respective statistical weights $g_{1}$ and $g_{0}$, the ionization energy $\epsilon$, the Boltzmann constant $k_{B}$, the Planck constant $h$ and the electron mass $m_{e}$. The main producers 
of electrons are species having the lowest ionization potential. In PP disks, such species are essentially alkali atoms (Sodium and Potassium), for which $\epsilon$ is of order $5 \mathrm{eV}$. Equation (2.30) can be written in term of their abundance relative to hydrogen. This gives the following expression for the electron fraction $x_{e}=n_{e} / n_{H}$ :

$$
x_{e}=\frac{\sqrt{2}}{\Lambda^{3} n_{H}} a^{1 / 2} \frac{g_{1}}{g_{0}} \exp \left(-\frac{\epsilon}{2 k_{B} T}\right),
$$

where $a=n_{K} / n_{H} \sim 10^{-7}$ is the abundance of alkali atoms (Umebayashi and Nakano, 1988). The important point to notice in the above equation is the overwhelmingly importance of the exponential (Balbus and Hawley, 2000). Indeed, the term $\exp \left(-\epsilon / 2 k_{B} T\right)$ respectively amounts to about $5 \times 10^{-7}, 3 \times 10^{-13}$ and $7 \times 10^{-26}$ when $T=2000,1000$ and $500 \mathrm{~K}$. This is a variation by almost 20 orders of magnitudes while the temperature only varies by a factor of four! Over the same range of temperature, the term multiplying the exponential only varies by a factor of a few. As a consequence of that exponential factor, the electron fraction $x_{e}$ equals $5 \times 10^{-7}, 10^{-13}$ and $10^{-26}$ respectively when $T=2000,1000$ and $500 \mathrm{~K}$. This simple estimate along with the considerations of the above section suggest that the MRI will be strongly affected by non-ideal MHD effects once the temperature drops below $1000 \mathrm{~K}$. In PP disks, this corresponds to all locations beyond a few tens of a AU from the central star (D'Alessio et al., 1998).

PP disks are dense The second reason for the small electron fraction in PP disks comes from their large gas density. The midplane number density of hydrogen molecules is of the order of $10^{14} \mathrm{~cm}^{-3}$ (see section 1), which should be compared to $\sim 1 \mathrm{~cm}^{-3}$ for the diffuse ISM or $\sim 10^{5} \mathrm{~cm}^{-3}$ for dense star forming cores (Mac Low and Klessen, 2004). This means that PP disks can hardly be ionized by external sources, like UV and X-ray photons or cosmic rays. Again, this can be recovered by the following simple calculation. Consider for simplicity the case of a pure gas disk (i.e. no dust particles) irradiated by a flux of cosmic rays. Free electrons are produced from molecular hydrogen at a rate $\xi$ :

$$
H_{2} \rightarrow H_{2}^{+}+e^{-}
$$

As discussed by Oppenheimer and Dalgarno (1974), the positively charged molecules $\mathrm{H}_{2}^{+}$reacts almost instantaneously with the numerous neutral $\mathrm{H}_{2}$ molecules to produce a series of molecular ions like $\mathrm{H}_{3} \mathrm{O}^{+}, \mathrm{HCO}^{+}, \mathrm{O}_{2}^{+}, \mathrm{H}_{3}^{+}$. These ions (collectively denoted $\mathrm{m}^{+}$in the following) dissociatively recombines with the free electrons with a reaction rate $\beta$ :

$$
m^{+}+e^{-} \rightarrow m
$$

These simple reactions translates into the following differential equation that describes the time evolution of the molecular ion number density:

$$
\frac{d n_{m^{+}}}{d t}=\xi n_{H_{2}}-\beta n_{m^{+}} n_{e^{-}}
$$


16Angular momentum transport during the formation and early evolution of stars - EES2012

Assuming electroneutrality in the fluid (i.e. $n_{m^{+}}=n_{e^{-}}$) and steady state gives

$$
x_{e}=\sqrt{\frac{\xi}{\beta n_{H_{2}}}} .
$$

The reaction rate for dissociative recombination is also given by Oppenheimer and Dalgarno (1974): $\beta \sim 3 \times 10^{-6} T^{-1 / 2} \sim 10^{-7} \mathrm{~cm}^{3} . \mathrm{s}^{-1}$ for a typical temperature of a few hundreds Kelvin. The ionization rate value is more debated. It is well known that galactic cosmic-rays have difficulties entering the inner solar system because of the solar wind (Gammie, 1996). The same is probably true of other young stars. At the same time, recent work suggests that the ambient ionization rate by cosmic rays in clusters (i.e. at the birth place of most stars) could be larger by three orders of magnitudes (Fatuzzo et al., 2006). A conservative estimate of the cosmic rays ionization rate based on present day measured values gives $\xi=10^{-17} \mathrm{~s}^{-1}$. When used in Eq. (2.35), one finds $x_{e} \leq 10^{-12}$ (the upper limit comes from the fact that the cosmic ray ionization rate is much reduced at the disk midplane, so our estimates is in fact an upper limit of the electron fraction). Again, this shows that the electron fraction is very small in PP disks and we can therefore anticipate that only the disk surface layers are sufficiently ionized for the MRI to become active.

The dead zone paradigm The simple calculations described above resulted in the dead zone paradigm proposed by Gammie (1996): the MRI can only develop in regions that are either hot enough $\left(T>10^{3} \mathrm{~K}\right)$ for thermal ionization to occur or in regions where cosmic rays (or other nonthermal ionization sources) can penetrate. In PP disks, the former condition is satisfied only close to the central star, at stellocentric distances smaller than a few tens of an AU. The second region (where nonthermal ionization is sufficient to activate the MRI) forms a thin layer at the disk surfaces. The MRI is expected to grow in both regions (and, as we shall see in the remaining of this lecture, the flow becomes turbulent there). In the disk midplane, the MRI is quenched and the flow remains laminar. The structure of the disk that results is illustrated in figure 4 . It can immediately be seen that such a disk structure is qualitatively different from standard $\alpha$ disk models. Although this has recently been challenged (Terquem, 2008), it was for example noted early on that such PP disks would have trouble evolving toward a steady state Gammie, 1996). This is because the accretion rate in the disk inner parts would be larger than the accretion rate that those active layers at the disk surfaces could sustained. This mismatch has led to a class of disk models that display an eruptive behavior, sometimes aimed at explaining the FU Orionis phenomenon (Zhu et al., 2010b] a; Martin et al., 2012).

It should be emphasized that the issue described above is serious and can compromise accretion onto young star in PP disks. The active layers at the disk surface can be so thin that their ability to sustain the observed inward mass flux is questionable. In addition, the magnetic field strength can reach equipartition at those dilute locations, stabilizing the MRI even if ideal MHD conditions prevail. 


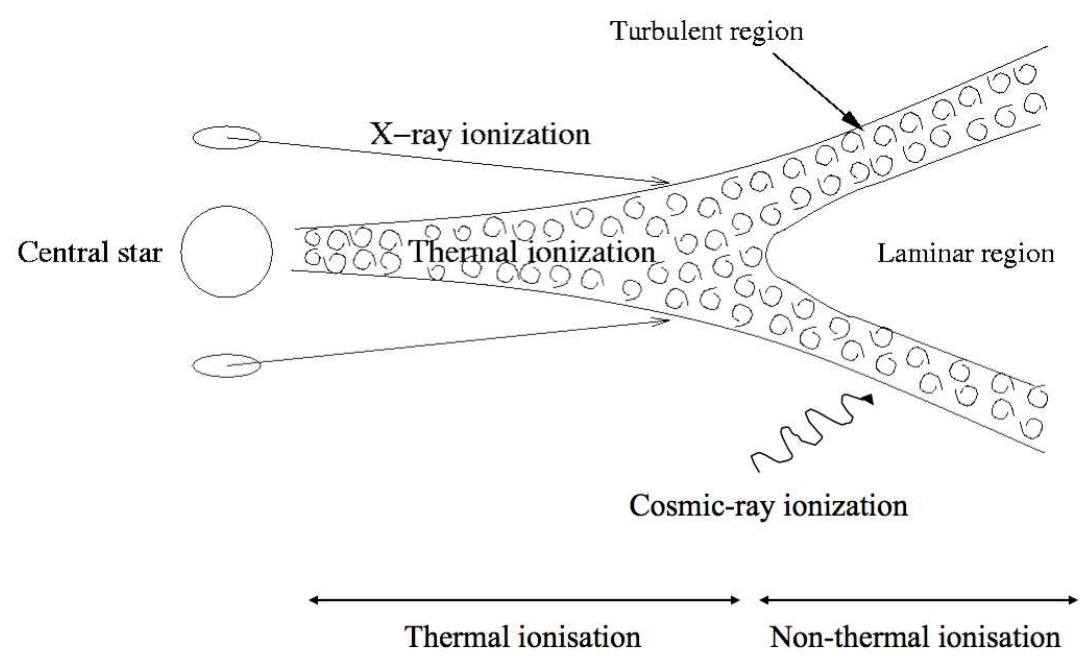

Fig. 4. Cartoon illustrating the state of PP disks as suggested by Gammie (1996). The innermost parts are MRI unstable as a result of thermal ionization which brings temperature above $10^{3} \mathrm{~K}$. In the disk inner parts, $0.1-1<R<10-20 \mathrm{AU}$, the disk is too cold for thermal ionization to be efficient and too massive for nonthermal ionization sources to produce enough free electrons all the way down to the disk equatorial plane. The MRI develops only in the disk upper layers where the ionization fraction is large enough as a result of $\mathrm{X}$-ray and/or cosmic rays ionization.

These are the reasons why people have built detailed chemical models of disks aimed at a precise determination of the electron fraction. Additional physical processes have been considered as potential solution to the problem, like X-ray irradiation (Glassgold et al., 1997), radioactive decay (Turner and Drake, 2009), far UV ionization (Perez-Becker and Chiang, 2011) or the effects of metal atoms (Fromang et al., 2002). The basic result that large portion of PP disks are immune to the MRI is robust. It becomes even worse when the effects of dust grains are considered. In the presence of dust grain (labeled "gr"), electrons can recombine through the reaction

$$
g r+e^{-} \rightarrow g r^{-} .
$$

The number of such reactions can be estimated as the product between the grains cross section $\sigma=a^{2}$, the electrons thermal velocity $u_{e}$ and the number density of grains (noted $n_{g r}$ ) and of electrons. When taking that reaction into account, Eq.(2.34) is modified and becomes, in steady state:

$$
\xi n_{H_{2}}-\beta n_{m^{+}} n_{e^{-}}-\sigma u_{e} n_{g r} n_{e^{-}}=0 .
$$

In the absence of grains, $n_{g r}=0$ and we recover the results of the preceding section. The key aspect of that equation is that the third term quickly dominates 
the second as soon as dust grains are introduced. If we assume that all the solids are in grains of size $a=10 \mu \mathrm{m}$ (in which case $\sigma \sim 10^{-6} \mathrm{~cm}^{2}$ ) and that the dustto-gas ratio is $10^{-2}$ in mass, then the dust fraction $x_{g r}=n_{g r} / n_{n} \sim 10^{-12}$. For temperatures of order $100 \mathrm{~K}$, we have $u_{e} \sim 10 \mathrm{~km} . \mathrm{s}^{-1}$. Putting things together, the ratio between the second and third terms of Eq. 2.37 can be calculated:

$$
\frac{\beta n_{m^{+}}}{\sigma u_{e} n_{g r}} \sim 10^{-7} \frac{x_{m^{+}}}{x_{g r}} \ll 1
$$

unless $x_{m^{+}}$is larger than $10^{-5}$, i.e. if the medium is completely ionized as far as the MRI is concerned. In the regime of small ionization fraction, the electron fraction is thus given by a balance between ionization and recombination on grains and writes:

$$
x_{e}=\frac{\xi}{\beta_{g r} n_{H_{2}}} .
$$

where we have introduced $\beta_{g r}=\sigma u_{e} x_{g r}=10^{-12} \mathrm{~cm}^{3} . \mathrm{s}^{-1}$. Using the same values as before for $\xi$ and $n_{H_{2}}$ in that equation, one obtains $x_{e} \sim 10^{-18}$. This is a significant reduction compared to gas phase chemistry. Formally, it can be traced to the absence of a square root in Eq. (2.39). This is because ion/electron recombination requires electrons colliding with ions, the number of which is proportional to that of electrons, while electron/grain recombination requires electrons colliding with grains, the number of which is independent to that of electrons.

The simple calculation above neglects several aspects of the problem, among which charge balance and charged grains reaction. It also assumes a single type of ions and a single grain size. It has nevertheless been confirmed in the past few years by detailed numerical integration of complex chemical networks (Ilgner and Nelson, $2006 \mathrm{~b}$ a; Bai, 2011b). The effect of dust grains is a severe problem for angular momentum transport in PP disks.

Ambipolar diffusion \& the Hall effect In fact, things are even more complicated than discussed above! This is because ohmic diffusion is not the only important non-ideal MHD effect in PP disks. Ambipolar diffusion and the Hall effect can be important as well. In chapter 1 of the book (see also Balbus, 2011), we derived the induction equation including all non-ideal terms. In the absence of dust grains, it writes

$$
\frac{\partial \boldsymbol{B}}{\partial t}=\boldsymbol{\nabla} \times\left(\boldsymbol{v} \times \boldsymbol{B}-\eta \nabla \times \boldsymbol{B}-\frac{\boldsymbol{J} \times \boldsymbol{B}}{n_{e} e}+\frac{(\boldsymbol{J} \times \boldsymbol{B}) \times \boldsymbol{B}}{\gamma \rho_{i} \rho c}\right),
$$

where, in addition to ohmic diffusion already discussed, see Eq.(2.26), the second term accounts for the Hall effect and the last term is due to ambipolar diffusion. It is possible to rewrite that equation in a form that is easier to interpret (Bai, 2011a):

$$
\frac{\partial \boldsymbol{B}}{\partial t}=\boldsymbol{\nabla} \times\left(\boldsymbol{v} \times \boldsymbol{B}-\frac{4 \pi \eta}{c} \boldsymbol{J}-\frac{4 \pi \eta_{H}}{c} \boldsymbol{J} \times \hat{\boldsymbol{B}}-\frac{4 \pi \eta_{A}}{c} \boldsymbol{J}_{\perp}\right),
$$




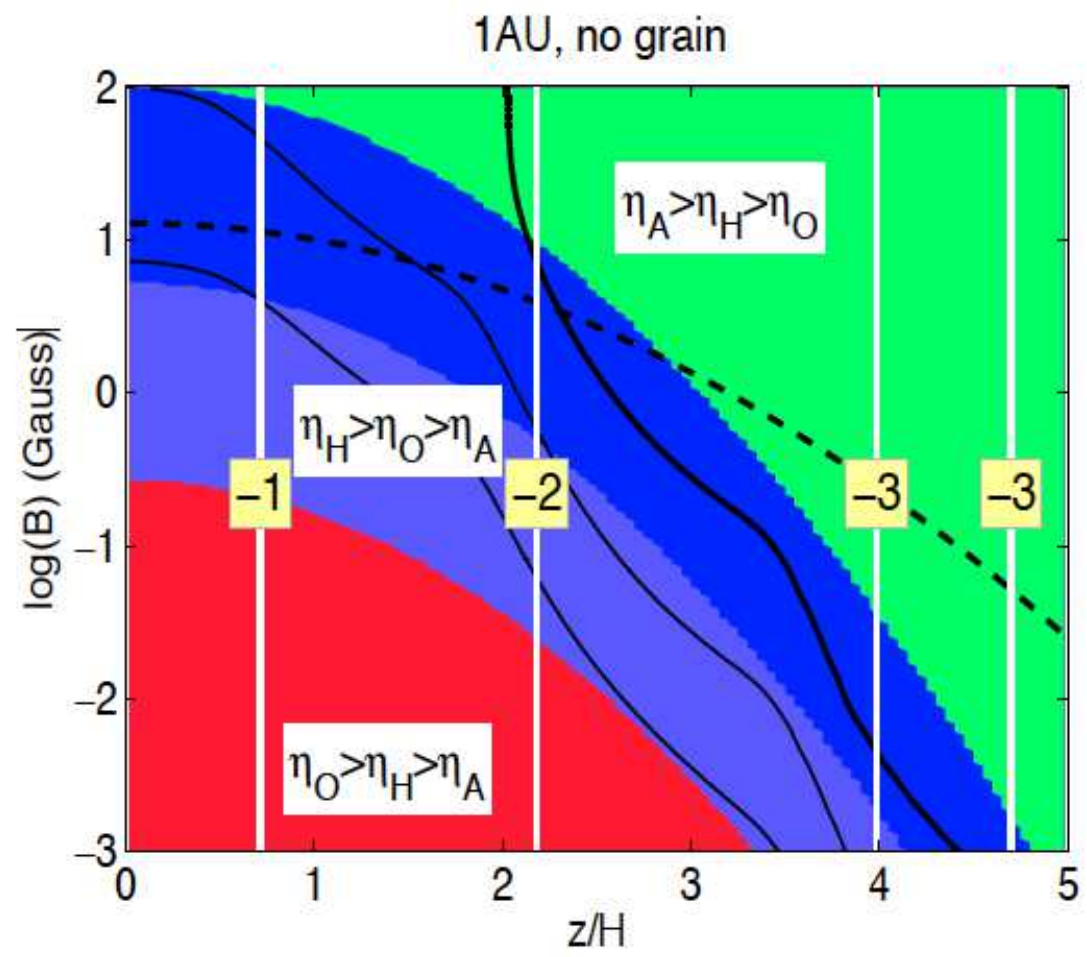

Fig. 5. Non-ideal MHD regimes (as a function of vertical position and magnetic field strength) for a typical PP disk model at 1 AU, assuming all dust grains have grown to form large particules. The red parts of the plot are dominated by Ohmic diffusion, blue parts are dominated by the Hall term while green parts are regions of the disk where ambipolar diffusion is the largest. From Bai (2011a).

where $\hat{\boldsymbol{B}}$ is a unit vector in the direction of $\boldsymbol{B}$ and $\boldsymbol{J}_{\perp}$ is the component of $\boldsymbol{J}$ that is perpendicular to $\boldsymbol{B}$. Order of magnitude estimates for $\eta_{H}$ and $\eta_{\boldsymbol{A}}$ can be obtained by comparing these two expressions:

$$
\begin{aligned}
\eta_{H} & \sim \frac{c B}{4 \pi e n_{e}} \\
\eta_{A} & \sim \frac{B^{2}}{4 \pi \gamma_{i} \rho_{i} \rho} .
\end{aligned}
$$

A similar expression for the Ohmic resistivity is provided by Eq. 2.29). The effect of ambipolar diffusion and the Hall term on the MRI are not straightforward. The former has been considered by Blaes and Balbus (1994) and the latter has been analyzed later on by Wardle (1999) and Balbus and Terquem (2001). The effect of the Hall term can be stabilizing or destabilizing depending on the rela- 
tive orientation between $\boldsymbol{\Omega}$ and $\boldsymbol{B}$. In the appropriate geometry, it is so strong that it has the potential to overcome the stabilizing effect of Ohmic diffusion (Wardle and Salmeron, 2012). Despite that complexity, one can guess from the form of the induction equation that the MRI is stabilized when the following Elsasser numbers associated with the effect of the Hall term or with that of ambipolar diffusion fall below unity:

$$
\begin{aligned}
\chi & =\frac{v_{A}^{2}}{\eta_{H} \Omega} \\
A m & =\frac{v_{A}^{2}}{\eta_{A} \Omega} .
\end{aligned}
$$

The expression given by Eq. (2.42) and (2.43) for the effect of the Hall and ambipolar resistivities can help get a feeling for the relative importance of both terms. In making these estimates, the strength of the magnetic field is very uncertain. Wardle (1997) suggests field strengths that range between $10^{-3}$ Gauss and $10^{2}$ Gauss, and we shall take the lower value of that range for illustrative puposes. Using values for the neutral density typical of PP disks midplane at $1 \mathrm{AU}, n_{H} \sim 10^{14}$ $\mathrm{cm}^{-3}$, as well as $\gamma_{i}=2.810^{13} \mathrm{~cm}^{3} \cdot \mathrm{s}^{-1} \cdot \mathrm{g}^{-1}, \rho_{i}=39 \rho$ (Balbus and Terquem, 2001) and a typical temperature $T \sim 100 \mathrm{~K}$, we can write

$$
\begin{aligned}
\eta & \sim\left(\frac{10^{3}}{x_{e}}\right) \mathrm{cm}^{2} \cdot \mathrm{s}^{-1}, \\
\eta_{H} & \sim\left(\frac{50}{x_{e}}\right)\left(\frac{10^{14} \mathrm{~cm}^{-3}}{n_{H}}\right) \mathrm{cm}^{2} \cdot \mathrm{s}^{-1}, \\
\eta_{A} & \sim\left(\frac{2.6 \times 10^{-3}}{x_{e}}\right)\left(\frac{10^{14} \mathrm{~cm}^{-3}}{n_{H}}\right)^{2} \mathrm{~cm}^{2} \cdot \mathrm{s}^{-1} .
\end{aligned}
$$

As can be seen, all of these resistivities scale like $x_{e}^{-1}$. In PP disks midplane at $1 \mathrm{AU}, n_{H_{2}} \sim 10^{14} \mathrm{~cm}^{-3}$ and ohmic resistivity dominates. The scaling $\eta_{H} \propto$ $n_{H}^{-1}$ and $\eta_{A} \propto n_{H}^{-2}$ suggests that the Hall term and ambipolar diffusion become more and more important in the disk upper layers. Indeed, at three scaleheights above the midplane 5, we have $\eta_{H} \sim 4.5 \times 10^{3} / x_{e} \mathrm{~cm}^{2} . \mathrm{s}^{-1}$ and $\eta_{A} \sim 21 / x_{e}$ $\mathrm{cm}^{2} . \mathrm{s}^{-1}$, thus $\eta_{H} \geq \eta \geq \eta_{A}$. At five disk scaleheight: 6 , we have $\eta_{H} \sim 1.3 \times$ $10^{7} / x_{e} \mathrm{~cm}^{2} \cdot \mathrm{s}^{-1}$ and $\eta_{A} \sim 1.9 \times 10^{8} / x_{e} \mathrm{~cm}^{2} . \mathrm{s}^{-1}$, thus $\eta_{A} \geq \eta_{H} \geq \eta$. This simple discussion demonstrates that we can expect PP disks midplane to be dominated by Ohmic resistivity, while the Hall effect and ambipolar diffusion should successively dominates the disk atmospheres. This order of magnitude estimate is confirmed by detailed calculations of the electron fraction that take into account a complex chemical network including dust grains. An example is given by the results of Bai (2011a) some of which are illustrated by figure 5. It confirms the results anticipated above: ohmic diffusion tends to dominate in the disk midplane, before

\footnotetext{
${ }^{5}$ Assuming the gas vertical profile to be Gaussian, $n_{H_{2}}(Z=3 H) \sim 1.1 \times 10^{-2} n_{H_{2}}(Z=0)$

${ }^{6}$ At $Z=5 H, n_{H_{2}}(Z=5 H) \sim 3.4 \times 10^{-6} n_{H_{2}}(Z=0)$
} 


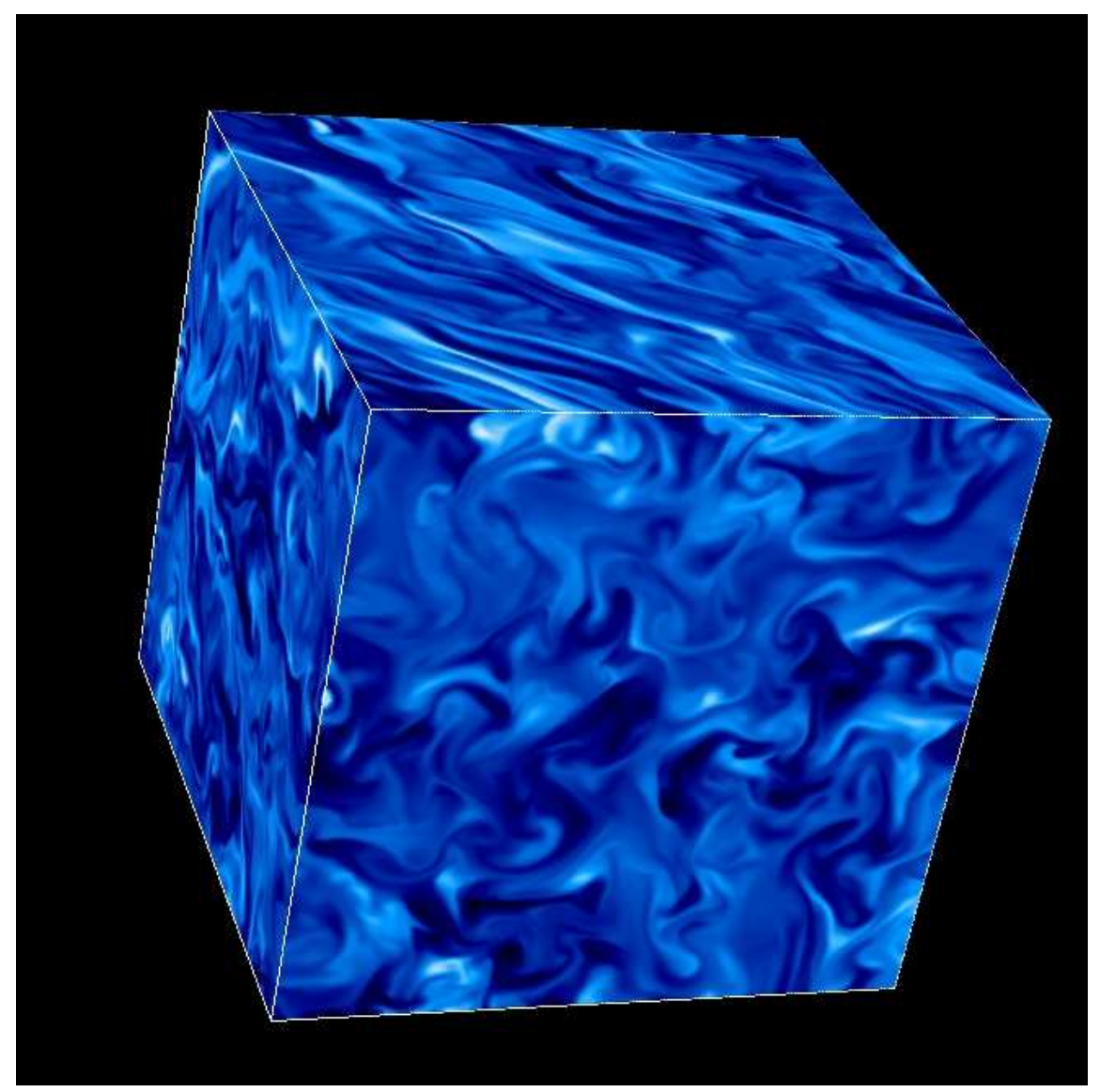

Fig. 6. Volume rendering illustrating the typical structure of the azimuthal component of the magnetic field in a typical MHD numerical simuations of the MRI performed in the idealized framework of the shearing box model (see section 3 for details). The flow is turbulent.

being successively replaced by the Hall effect and ambipolar diffusion in the disk upper layers. Although figure 5 is a plot of the disk structure at 1 AU from the central star, the outer disk retain the same qualitative properties. We will come back to the consequences of these non-ideal terms during the nonlinear evolution of the MRI in section 3.2 and 4.1

\section{MHD turbulence}

So far, we have focused only on the linear aspects of the MRI. But of course, as it grows, nonlinear terms in the MHD equations start to influence the flow. Its prop- 


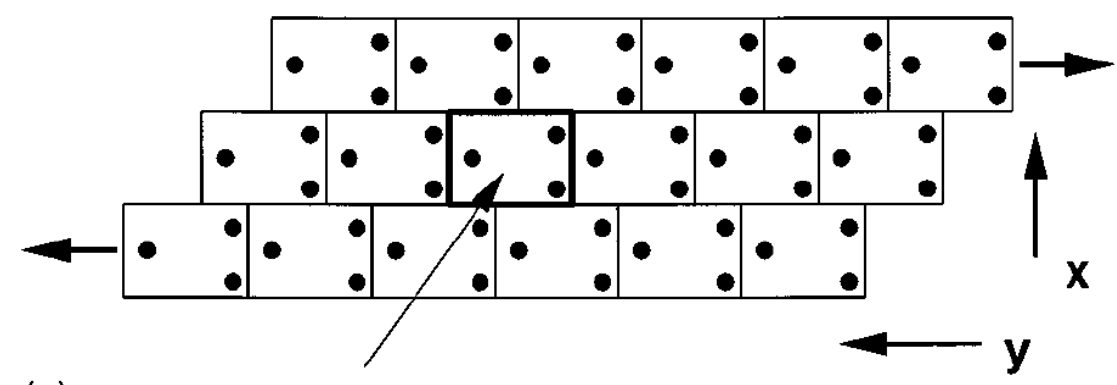

(a)

Computational Domain

Fig. 7. Cartoon illustrating the origin of the shearing box radial boundary conditions. The idea is that the disk is composed of many such boxes, statistically identical, that are sliding past each other because of the background orbital shear. Adapted from Balbus and Hawley (1998).

erties during that stage are best studied with the help of numerical simulations. In this section, we review the results that have been established using such simulations in the last twenty years. The focus of this section is on idealized numerical experiments such as shown in figure 6 that aim at understanding MHD turbulence as an angular momentum transport process. This is a neccessary first step before building realistic models of protoplanetary disk structure and examining the consequences of the MRI for planet formation models.

\subsection{Methods and early results}

The simultaneous discovery of the MRI and the rise in computing power witnessed during the early 90's quickly established a series of results that demonstrated the tremendous potential of that instability. In this section, we briefly describe the methods that were developed to that end before summarizing the most important of these findings.

The shearing box model As discussed in section 2.1, the physics of the MRI is easier to understand if the analysis is made local. That local approach has proved very useful ever since the discovery of the MRI. It is now known as the shearing box model. It helps to focus on the dynamics of the flow without the burden of having to consider the entire disk (with additional difficulties such as ill-posed boundary conditions for example). This is of course at the cost of the realism of the approach, but the shearing box model has led to spectacular progress (and still does!). What is true for the analytical analysis remains true when developing numerical simulations of the MRI. The set of equations that is used in this case is derived from the MHD equations as described in section 2.1. There are two 
possible variants: one is to stick to the analysis detailed there and solve Eq. (2.3), (2.4) and (2.5). But, as we have seen, the fluid perturbations associated with the channel modes of the MRI are incompressible. An alternative is thus to use the incompressible MHD equation (the continuity equation then reduces to the constraint $\boldsymbol{\nabla} \cdot \boldsymbol{v}=0$ ) in a rotating frame. The Coriolis force and the tidal potential discussed in section 2.1 remain unchanged. This second approach has recently been adopted by some authors (Umurhan and Regev, 2004; Lesur and Longaretti, 2007). However, historically, most authors used the compressible formulation of the MHD equations to investigate the nonlinear evolution of the MRI. This is probably due to the rise of computational astrophysical fluid dynamics that occurred simultaneously to the discovery of the MRI and to the fact that astrophysical flows are highly compressible in most instances. Thus versatile codes developed to address astrophysical fluid dynamics problems are preferentially solving the full set of compressible MHD equations.

There are nevertheless aspects of the problem that are common to both approaches. One such aspect is the boundary conditions. To understand them requires to put the shearing box model in its wider context: the idea is that the entire accretion disk is filled with many such boxes, with the important point that the statistical properties of the flow is the same for all boxes. This is valid, for example, in the case of a turbulent flow whose characteristic scale is smaller than that of the box. This geometrical picture is illustrated in figure 7 . The many boxes that constitute the disk are sliding past one another during a simulation. Thus periodic boundary conditions are adopted in the azimuthal and vertical directions. The radial boundary conditions are more complicated, though. Consider for example an observer sitting on the inner side (in the radial, or $\mathrm{x}$, direction) of the box at a azimuthal location. As time goes on, the observer sees the outer radial side of neighboring boxes (located closer to the central object) sliding past him. Because all these boxes are statistically identical, the flow it sees in these boxes at any given time is the same as the flow in its own box, but at the outer radial side of its box, and located at a position in $y$ that varies periodically with time. As we see, the radial boundaries conditions are still periodic but in a peculiar, time varying sense. This is called shearing periodicity. It has first been introduced in numerical simulations by Hawley et al. (1995) and the interested reader is referred to that paper where the mathematical formulation of the shearing box boundary conditions is given.

Numerical investigations of the nonlinear evolution of the MRI was quickly undertaken in the early 90's by several teams. Hawley and Stone (1995) and Hawley et al. (1995) pioneered the way by extending the newly developed algorithm of ZEUS (Stone and Norman, 1992a, b) that uses a stable operator split technique. Brandenburg et al. (1995) used a sixth-order finite difference scheme that was a precursor to the now well-known Pencil Code7 7. There are now several different codes in addition to ZEUS and the PEnciL Code that can be used to solve the shearing

\footnotetext{
${ }^{7}$ See http://www.nordita.org/software/pencil-code
} 


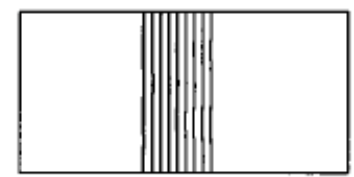

1.46

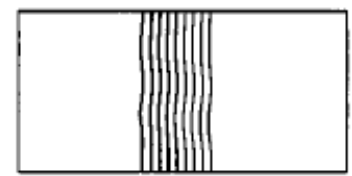

1.70

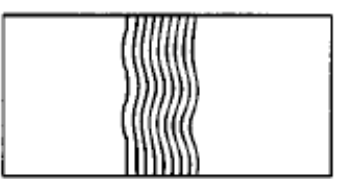

1.95

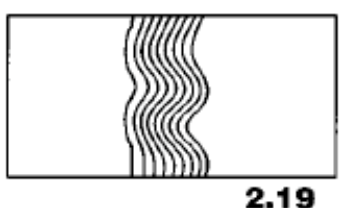

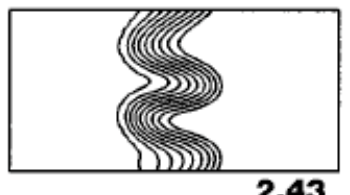

2.43
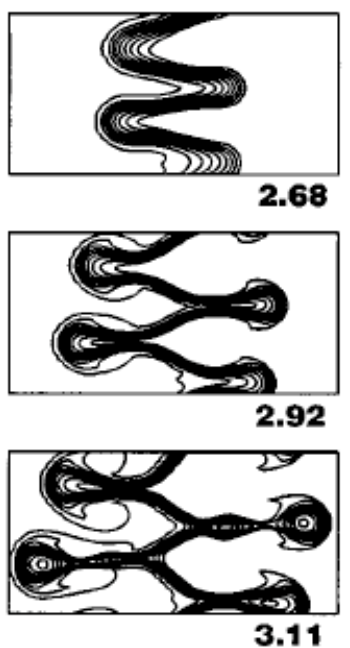

Fig. 8. Early evolution of poloidal magnetic field lines in an accretion disk in the ideal MHD limit. The MRI quickly distorts the initially vertical field. Called channel modes, this perturbation produces fingers of field and matters that propagate radially. Toward the end of the evolution $(t=3.11)$, the channel mode amplitude becomes nonlinear and vulnerable to parasitic instabilities, as first discussed by Goodman and Xu (1994). Time is labelled in orbits under each panels. From Balbus and Hawley (1998).

box equations. Many of them use finite volume schemes based on the Godunov method (Toro, 1997). This is the case of ATHENA (Stone et al., 2008), PLUTO (Mignone et al., 2007), NIRVANA-III (Ziegler, 2004, 2008) or RAMSES (Tevssier, 2002; Fromang et al., 2006). Also publicly available is the pseudo-spectral code SNOOPY 8 that solves the incompressible MHD equations. Such a wealth of different numerical methods means that published results can now be carefully tested and are more robust.

Transition to turbulence The first numerical work related to the MRI appeared as a companion paper to the analytical derivation of its dispersion relation. Hawley and Balbus (1991) presented 2D numerical simulations that backed

\footnotetext{
${ }^{8}$ See http://ipag.osug.fr/ glesur/snoopy.html
} 
up and extended their linear calculation. In particular, they confirmed the properties of the channel modes discussed above. The structure of the magnetic field lines resulting from the evolution of an initially pure vertical field are illustrated on figure 8. The oscillating fingers due to the channel modes create strong magnetic field that moves radially in the disk, producing large vertical velocity gradients and strong currents. Both are prone to Kelvin-Helmotz like instabilities, as investigated in detail by Goodman and Xu (1994). These "parasitic" instabilities grow on top of the regular pattern associated with the MRI linear modes, disturb the flow and provide a pathway to MHD turbulence.

The road to MHD turbulence The transport properties resulting from the nonlinear development of the MRI can be quantified by measuring the stress tensors discussed in the introductory chapter of this book. As detailed there, the later is the sum of the Maxwell and Reynolds stresses. To make connection with the standard $\alpha$-disk theory presented in section 10 it is convenient to normalize its value by the thermal pressure, so that the parameter $\alpha$ can be measured in numerical simulations according to the relation:

$$
\alpha=\frac{\left\langle T_{R \phi}^{t u r b}\right\rangle}{\langle P\rangle}=\frac{\left\langle-B_{R} B_{\phi}+\rho v_{R} \delta v_{\phi}\right\rangle}{\langle P\rangle} .
$$

Early simulations performed in the 90's solidly established some key results that still hold today. The first were derived using the homogeneous shearing box threaded by a uniform magnetic field (Hawley and Balbus, 1992; Hawley et al., 1995):

- When the dissipation coefficients are small enough not to affect its linear stage, the nonlinear development of the MRI always leads to MHD turbulence that transports angular momentum outward.

- The turbulence is subsonic and the Maxwell stress dominates the Reynolds stress by a factor of a few.

- The value of $\alpha$ ranges from $10^{-3}$ to a few times $10^{-1}$ depending on the magnetic field strength. $\alpha$ is an increasing function of the mean magnetic energy.

- The above results hold if the magnetic flux is azimuthal instead of vertical, even if $\alpha$ is slightly smaller in that case.

These results were obtained in the presence of a nonzero magnetic flux (either vertical or azimuthal). In the shearing box, such a flux is conserved during a simulation because of the periodic boundary conditions. An interesting limit of that case is that of a vanishing magnetic flux. This was first considered in the homogeneous shearing box by Hawley et al. (1996). Starting their simulations with a random magnetic field, they also found a robust breakdown of the flow into MHD turbulence. The rate of angular momentum transport was found to be weaker, though, with $\alpha \sim 10^{-2}$, independent of the field strength. It is important to realize that this case is qualitatively different from the so-called net flux case. Indeed, their is no linear instability as such in this situation. In addition, a dynamo mechanism is needed to sustain the turbulence over many dynamical timescales. 
Otherwise, any finite dissipation will force the flow back to a laminar field as the magnetic field gradually disapears. The dynamo mechanism operating in accretion disks was out of the scope of the paper of Hawley et al. (1996) but one of their most important finding was that the standard kinematic dynamo theory was inadequate to describe the field amplification mechanism: removal of the magnetic feedback on the flow by the Lorentz force always leads to the decay of the turbulence.

The simulations discussed so far were obtained in the framework of the homogeneous shearing box, neglecting the vertical stratification of density. This limitation was soon alleviated in two papers by Brandenburg et al. (1995) and Stone et al. (1996) who studied the development of the MRI in stratified shearing boxes. Both studies considered an initial magnetic field configuration with vanishing mean vertical magnetic field. Both found that the MRI leads to vigorous outward angular momentum transport with typical $\alpha$ values in the range $5 \times 10^{-3}$ to $10^{-2}$. The disk was found to develop a structure composed of two parts:

- A weakly magnetized layer around the disk midplane $(|Z| \leq 2-3 H)$, where thermal pressure dominates over magnetic pressure and with subsonic velocity fluctuations

- Two magnetized layers that form the disk atmosphere $(|Z| \geq 2-3 H)$ where thermal and magnetic pressure are comparable and with sonic velocity fluctuations.

The case of stratified shearing boxes in the presence of a net vertical field was considered soon after by Miller and Stone (2000). It proved more problematic. As in homogeneous boxes threaded by vertical magnetic fields, channel modes were found to grow to large amplitude. Magnetically dominated regions appeared and were expelled from the computational box, along with most of the disk mass, because of magnetic buoyancy. This is in contrast with homogeneous boxes in which no such vertical gradient is present and where the channels themselves are destabilized by parasitic instabilities and turn turbulent. In stratified boxes, the disk is never able to reach a quasi steady state that transports angular momentum outward. Because of these difficulties in finding a gentle turbulent state, this configuration was left aside from mainstream research for about a decade. This is a pity as this is a natural magnetic field configuration in PP disks (in which the net magnetic field is believed to be the remnant of the magnetic field that was threading the dense core out of which the young star formed). We shall return to that important problem in section 4.1 .

\subsection{Saturation of MHD turbulence: idealized simulations}

MHD turbulence in the ideal MHD limit The early simulations described above were done mostly in the limit of ideal MHD, i.e. neglecting all dissipative terms in the equations. This was made possible by using numerical schemes that are stable when solving the Euler fluid equations. As computer power increased, larger resolution became available and convergence studies became possible. A problem soon emerged: in a series of papers devoted to the case of the homogeneous shearing box in the absence of a net magnetic field, Fromang and Papaloizou 


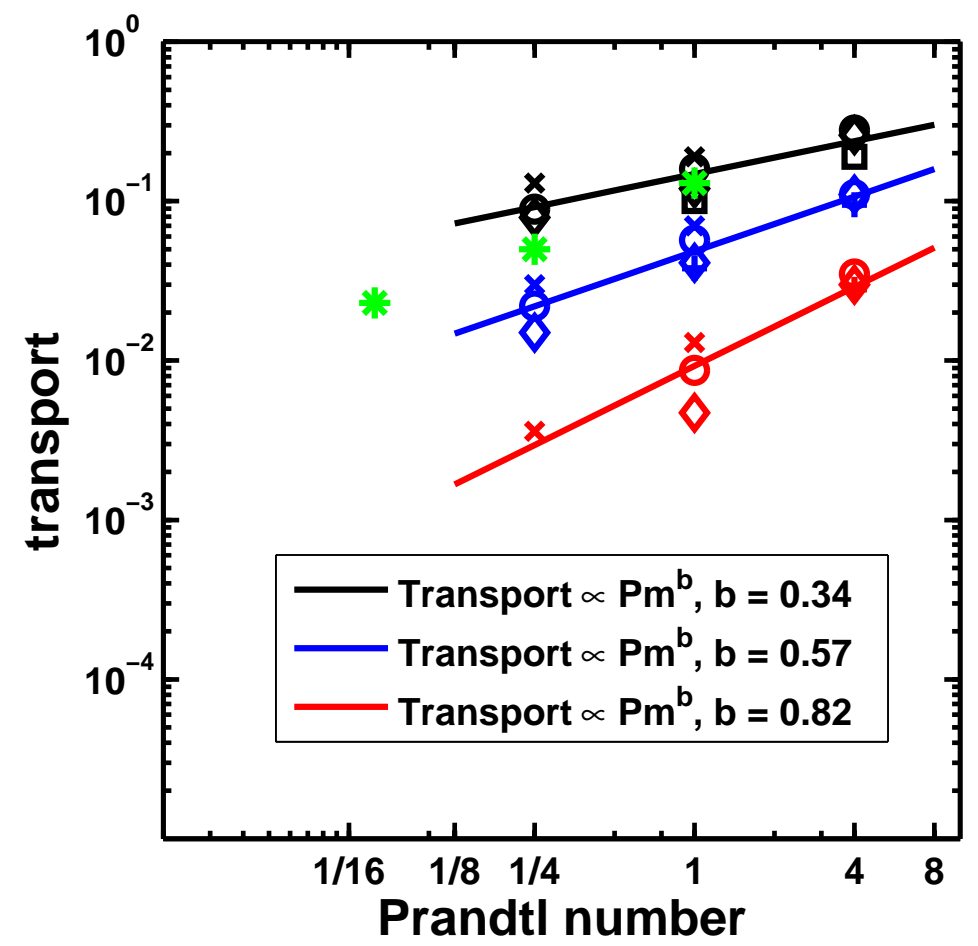

Fig. 9. Rate of angular momentum transport (as measured using the $\alpha$ parameter, see text for details) as a function of the magnetic Prandtl number Pm measured using the homogeneous shearing box model and in the presence of a vertical magnetic field. Red, blue and black symbols correspond to plasma beta $\beta=10^{4}, 10^{3}$ and $10^{2}$, respectively. The different symbol correpond to different Reynolds numbers: $R e=400$ (squares), 800 (plus), 1600 (diamonds), 3200 (circles) and 6400 (crosses). The green stars are high resolution runs for which $R e=c_{0} H / \nu=20000$ and $\beta=10^{3}$. Finally, the lines are power law fits for each values of $\beta$. From Longaretti and Lesur (2010).

2007); Pessah et al. (2007); Simon et al. (2009); Guan et al. (2009); Bodo et al. (2011) all found, by solving the set of ideal MHD equations, that $\alpha$ was a decreasing function of resolution. Since the only difference in each sequence of simulation was the size of the grid cells, these results demonstrated that the nature of the flow at that small scale affects its large scale structure. In other words, there is not enough room to separate the small dissipative scales of the flow from its large and astrophysically relevant scale. Both scales talk to each other. This may not be the case in real systems, but was definitely the case in these simulations. The worrying aspect of the simulations like that of Fromang and Papaloizou (2007) is that the structure of the flow at the cells scale is strongly affected by the details 
of the numerical scheme. This means in turn that the numerical scheme influences the flow largest scale. To solve that problem, a proper treatment of the small scales is mandatory. This was dealt with by including physically motivated dissipation coefficients in the simulations. That small modification to the equations soon lead to new results as we shall see below.

Before we move on, though, we should mention that the convergence issue described above has now been examined for different field configurations. It was found that $\alpha$ values are converged in the presence of a mean toroidal (Guan et al., 2009) or vertical (Simon et al., 2009) magnetic field when the resolution is varied. The reason for the difference with the zero net flux case is still unclear but might be due to the presence of a net flux that constantly help the MRI be reactivated. The convergence issue of MRI-induced MHD turbulence was also considered in stratified boxes (Davis et al., 2010; Shi et al., 2010). Despite having zero net vertical and toroidal flux, their simulations display nicely converged $\alpha$ value when the resolution increases. The difference with unstratified shearing boxes is again not understood, and might be tied to the existence of a large scale density gradient in the vertical direction that enables magnetic buoyancy to play a role in the dynamics.

The role of the magnetic Prandtl number The numerical convergence issues described above lead to a series of systematic study of the dependence of the turbulence properties on small scale dissipation coefficients. As a first step these studies focused on the effect of a kinematic viscosity $\nu$ and ohmic resistivity $\eta$. The most robust result that was obtained is that the rate of angular momentum transport is an increasing function of the magnetic Prandtl number Pm defined according to the relation

$$
P m=\frac{\nu}{\eta}=\frac{R m}{R e}
$$

where $R m=c_{0} H / \eta$ is the magnetic Reynolds number of the flow. This behavior had been speculated quite early on (see for example the concluding section of Balbus and Hawley, 1998) but could not be studied in the 90's because of limited computational resources. It is in fact very robust: it was observed in the absence of a net magnetic flux (Fromang et al., 2007), in the presence of a net magnetic flux in the azimuthal direction (Simon and Hawley, 2009) and in the presence of a net magnetic flux in the vertical direction (Lesur and Longaretti, 2007; Longaretti and Lesur, 2010). For illustrative purposes, figure 9 summarizes the results of that last study as it presents the most extensive parameter space coverage. These first papers of the $P m$-effect were devoted to the case of the homogeneous shearing box, but Simon et al. (2011) then showed that the same trend exists, albeit weaker, when vertical stratification is taken into account. Their simulations, however, were done for the zero net flux case. It also covered a limited region of parameter space because of the large computational cost of such stratified simulations. Future work is needed. 
Ambipolar diffusion and the Hall effect The magnetic Prandtl number effect is definitely robust, but it might be irrelevant in the PP disks context. Indeed, as shown in the previous sections, the disk locations where ohmic diffusion is the dominant dissipative term in the induction equation are located well inside the dead zone where the MRI is stabilized. In turbulent parts of PP disks, the dominant dissipative term are ambipolar diffusion and the Hall effect. The effect of ambipolar diffusion has been considered in details by Hawley and Stone (1998), Bai and Stone (2011) and Simon et al. (2013). Their work confirms and extents the results of linear analysis: the maximum $\alpha$ value is found to drop significantly as $A m$ falls below unity. In addition, the larger the value of $A m$, the weaker the magnetic field needs to be for the MRI to operate (with consequently weaker turbulent transport).

The case of Hall diffusion is more difficult to handle numerically. This is because of the presence of a new kind of motion, the whisler waves, that put strong constraints on the timestep of the calculation. Sano and Stone (2002a, $\mathrm{b}$ ) published early simulations of the MRI in that regime that confirmed the linear analysis but did not find any significant effect of the Hall term during its nonlinear stage. However, as recently pointed out by Wardle and Salmeron (2012), the too small Hall term they used in their simulations makes a definite interpretation of their results quite difficult. Additional work is needed that focuses on the relevant regime.

Current status, open questions While some results are now firmly established, it is fair to say that we still lack a complete understanding of the saturation of MRI-induced MHD turbulence as a function of the disk parameters. Below is a list of some of the most critical questions and, when possible, the immediate prospect for their solutions:

- The sensitivity of angular momentum transport to $P m$ immediately raises the question of the asymptotic behavior of the flow in the small $\mathrm{Pm}$ limit that is relevant for $\mathrm{PP}$ disk 9 . The best simulations are currently addressing that question, but some hints are already present in the literature: for example, in the case of a pure toroidal magnetic field, figure 7 in Simon and Hawlev (2009) shows using blue symbols a sequence of $\alpha$ values computed for $R m=3200$ and decreasing values of $P m$ (or, equivalently, increasing $R e$ ). Clearly, it is tempting to say that $\alpha \rightarrow 0.02$ when $P m \rightarrow 0$. Similarly, in the case of a vertical magnetic field, figure 9 shows early suggestions of convergence: the series of runs having $R m=1600$ and $\beta=10^{3}$ (blue and green colors) appears to converge toward $\alpha=2 \times 10^{-2}$ at low Pm. Whether this trend is confirmed at much lower Pm and its dependence on the value of $R \mathrm{~m}$ should be the focus of future work, but current results indicate that transport at low $\mathrm{Pm}$ is possible in the presence of a net flux.

\footnotetext{
${ }^{9}$ We have seen in section 1 and 2 that the electron fraction can be extremely small. As a result, the resistivity is much larger than the viscosity in PP disks and the magnetic Prandtl number is much smaller than unity
} 


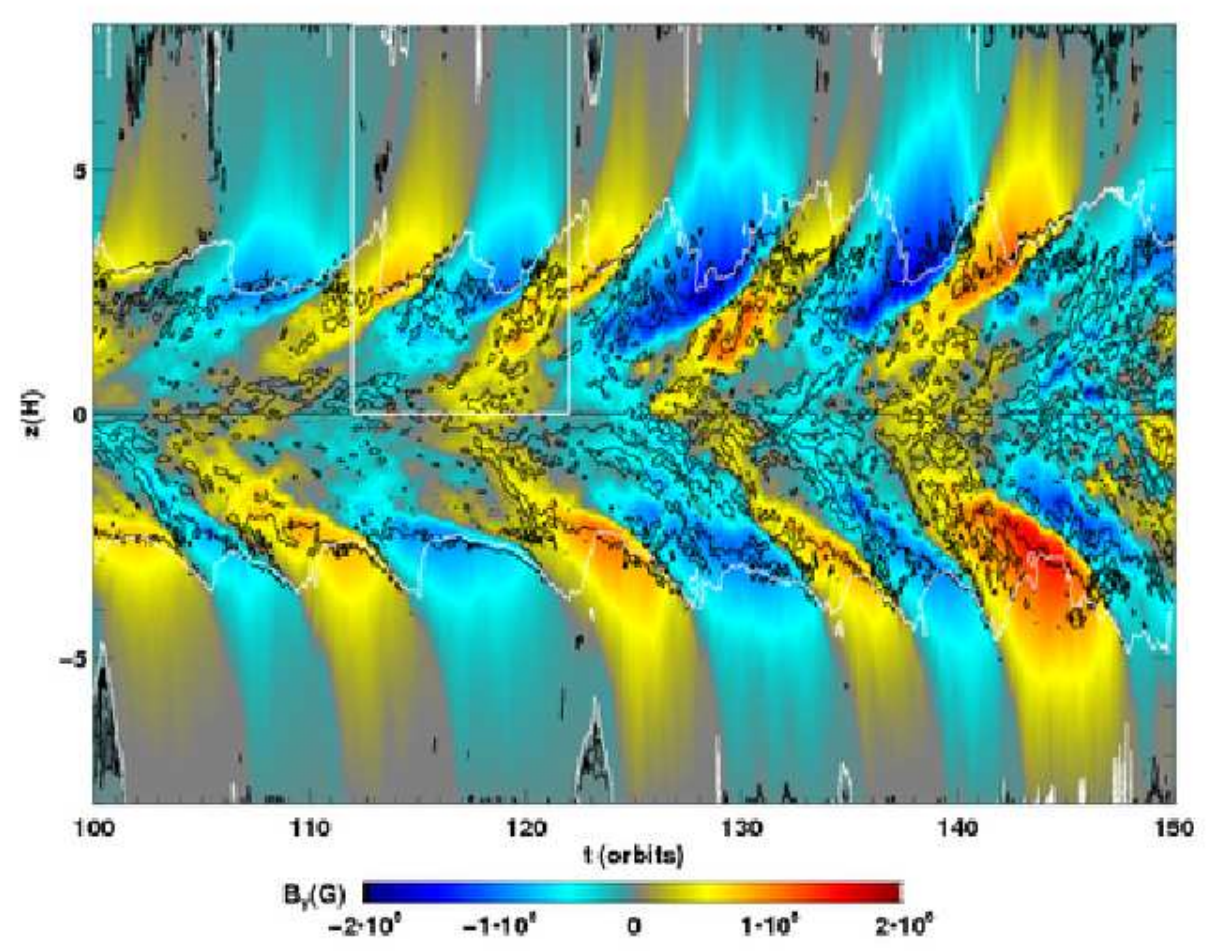

Fig. 10. Spacetime diagram showing the evolution of the horizontally averaged azimuthal magnetic field vertical profile in a typical stratified shearing box simulations in the absence of a vertical field. A nonzero mean toroidal field is produced in the disk midplane before being expelled toward the disk surface layer in a quasi periodic way. This typical plot has been named the butterfly diagram and is believed to be a manifestation of some sort of mean field dynamo. From Shi et al. (2010).

- In the absence of a net flux, the $P m$ effect is spectacular: when $P m<1$, the turbulence dies off and the flow return to its laminar state (Fromang et al., 2007). This is not without similarities with the known results that small scale dynamo in incompressible fluids is more difficult to trigger (Schekochihin et al., 2004) at low Pm: in that limit, there exists a critical magnetic Reynolds number $R m_{c}$ above which small scale dynamo is observed to grow that increases as $P m$ decreases (Iskakov et al., 2007). Is it the same for the MRI? What are the properties of the dynamo mechanism that operates in this regime? The computational requirements to address that problem are massive, and it is not clear that brute force is the answer. Recently, Herault et al. (2011) proposed a novel approach to the problem and found that dynamo cycles exist even when dissipation coefficients are large. Whether the properties of such cycles are 


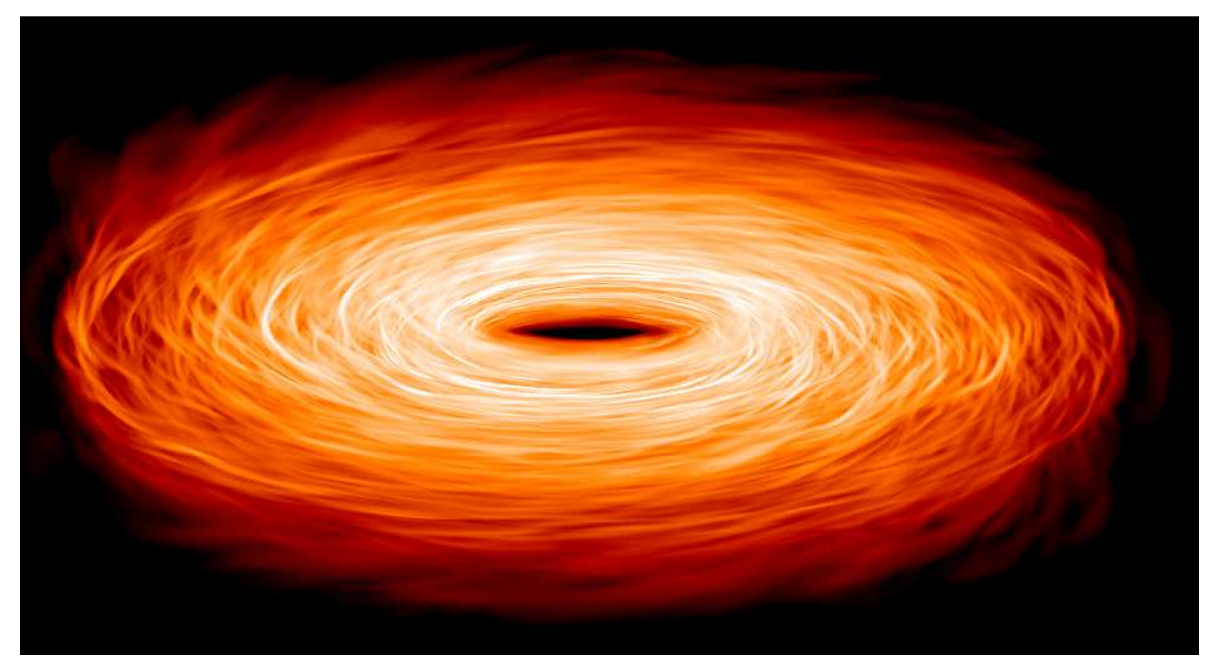

Fig. 11. Snapshot of the magnetic pressure produced in a typical MHD global simulation of a turbulent PP disk. Simulations data have been post-processed using the code RADMC (http://www.ita.uni-heidelberg.de/ dullemond/software/radmc-3d/) to produce the $3 \mathrm{D}$ rendering. Image courtesy Mario Flock.

imprinted into fully developed MHD turbulence remains to be demonstrated, but the results appear promising at this stage.

- The influence of density stratification on these results is a third important open issue. Several papers reported the appearance of a mean toroidal field, the strength of which is modulated in time (Brandenburg et al., 1995; Gressel, 2010; Shi et al., 2010; Davis et al., 2010; Simon et al., 2012). This mean magnetic field is expelled toward the disk upper layers producing a regular pattern in the spacetime diagram (see figure 10). This properties of the flow has been named the butterfly diagram. The reason for its appearance is not firmly established and its potential role in the dynamo mechanism is still debated.

\subsection{Global disk simulations of turbulent PP disks}

So far the discussion has been restricted to local studies, both analytically in our derivation of the stability criterion as well as numerically in our summary of the most salient results of shearing boxes numerical simulations. However, PP disks are large scale objects in which the key parameters (surface density, temperature, ionization fraction, magnetic flux) potentially evolve with position and time. However useful a local approach is to our understanding of the turbulence properties, we ultimately want to compare these models with observations of PP disks as a whole. This is why several teams developed global numerical simulations of turbulent accretion disks. 
The issue of resolution The rule of thumb when discussing global simulations of PP disks is that the numerical resolution of the calculation is always too low! Ideally, one would want to make a model of a disk covering the planet formation region, say from 1 to $10 \mathrm{AU}$ of the central star and covering a few vertical scaleheights on both sides of the disk midplane (say, $\pm 5 \mathrm{H}$ to fix ideas). Typical PP disks have disk aspects ratio $H / R$ that range from about 0.05 to 0.1 . For the thicker value (which is easier to handle numerically), this means the simulations would cover about 10 scaleheights in the vertical direction and 25 scaleheights in the radial direction. Modern day numerical simulations have a resolution of a few hundreds cells in each direction. This means that the most resolved global simulations today can at best achieve a resolution of 10 to 20 cells per scaleheight. This should be contrasted with the resolution of about 128 to 256 cells per scaleheight used in local simulations to study the saturation properties of the turbulence as a function of microscopic dissipation coefficients. Clearly, it is impossible at present time to properly include dissipation in global simulations and be in a parameter regime relevant for PP disks. One should instead rely on numerical dissipation to stabilize the numerical scheme when performing such simulations, and hope for the best...

Typical models and main results Due to the limitations detailed above, global simulations of turbulent PP disks still face drastic limitations even when resolution is reduced to its minimum acceptable values. Simplifications have to be made. One of them is an oversimplified thermodynamics. Since PP disks are mostly passive disks heated by their harboring star, a locally isothermal equation of state is often used. For simplicity, the gas sound speed obeys a power law

$$
c_{s}^{2}(R)=c_{0}^{2}\left(\frac{R}{R_{0}}\right)^{q}
$$

in most published simulations. $q=-1$ is often adopted. Another limitation is provided by the limited radial extent of the simulations. Published simulations usually cover a radial extent of about a decade. This means that radial boundaries (the treatment of which is not without problem but is beyond the scope of this lecture) are never far from the bulk of the disk. They might influence the flow properties. This is a potential problem that should be kept in mind. Another difficulty with global simulations is their large range in dynamical timescales: the orbital time is much larger at the outer edge than at the inner edge. This means that the simulations requires to be evolved for many orbital period at the inner edge before reaching a quasi steady state at the outer edge. This is not without consequences in terms of computing resources needed for such simulations.

Despite these difficulties and caveats, papers dedicated to studying the structure of the flow in turbulent PP disks by means of global simulations have been published by several authors. The first simulations were performed in the cylindrical limit (Steinacker and Papaloizou, 2002; Papaloizou and Nelson, 2003; Winters et al., 2003; Sorathia et al., 2012). They were built as an extension of the first pioneering 
simulations of Armitage (1998) and Hawley (2001) that were more specifically aimed at studying black hole accretion. Recent work also includes vertical stratification (Fromang and Nelson, 2006; Sorathia et al., 2010; Beckwith et al., 2011;

Flock et al., 2011; Fromang et al., 2011). An example of the results of such global simulations is given in figure 11] The main results are:

- Typical $\alpha$ values are in the range $5 \times 10^{-3}$ to a few $10^{-2}$, consistent with shearing boxes simulations performed at the same resolution and with the same B-field configuration.

- The results appears to display numerical convergence for resolution of 32 cells per $H$ or higher (Sorathia et al., 2012).

- There is a correlation between the local stress tensor and the local vertical flux of magnetic field (Sorathia et al., 2010; Beckwith et al., 2011)

- There are significant turbulence fluctuations at scales larger than the typical disk scaleheight (Beckwith et al., 2011; Flock et al., 2012). As a result, a minimum azimuthal size of the computational box of $\sim \pi / 2$ is required.

- The existence of meridional circulation seems to be excluded in fully turbulent disks (Flock et al., 2011; Fromang et al., 2011), which is a significant departure from the expectations of viscous disk theory.

Before closing this section, however, it is important to stress that most global simulations published so far have assumed ideal MHD, with effectively zero net vertical flux. This is a significant limitation compared to the wide range of configurations that have been probed using shearing boxes simulations. Much work remains to be done to improve the quality of global simulations of PP disks.

\section{Consequences for protoplanetary disks and planet formation}

Sections 2 and 3 summarize the understanding we have gained during the last couple of decades about MRI-driven angular momentum transport in PP disks. The situations considered in these sections are rather academic: idealized situations are constructed with the aims to understand the fundamental properties of the MRI and its nonlinear consequences. The next stage is of course to build realistic models of PP disks based on these findings. Such models can then be used to refine our understanding of planet formation scenarii. The purpose of the present section is to review these topics and to highlight some of the outstanding problems that are still to be solved.

\subsection{Protoplanetary disks structure}

Layered accretion Since the seminal paper of Gammie (1996), layered accretion is the classical paradigm describing the flow in PP disks: it consists in a fully turbulent inner disk, while at larger radii, the flow is laminar in the bulk of the disk and turbulent in its surface layers. Local simulations have investigated the z-dependence of the flow structure at radii where a dead zone is present while global simulations have focused on the dynamics taking place at the inner edge of the dead zone. The main results of these simulations are: 
- Turbulent motions in the active surface layers of the disk excite waves that propagates deep into the dead zones (Fleming and Stone, 2003). These waves create a sustained angular momentum transport due to the associated Reynolds stress. Typical $\alpha$ values that can be attributed to such waves are of the order of $10^{-5}$ to $10^{-6}$ and depend on the dead zone mass.

- Most of the published simulations are performed using an isothermal equation of state. This is not appropriate for the optically thick inner parts of the disk, where cooling is slow and heating is due to the dissipation of turbulent energy. Some authors (Hirose and Turner, 2011; Flaig et al., 2010, 2012) recently relaxed that approximation in stratified shearing boxes calculations. They reports quantitative changes only, the qualitative picture of layered accretion being unaltered. However, recent mean field models of the coupling between dynamics and thermal processes suggest that new effects might affect the global structure of the disk (Latter and Balbus, 2012). The confirmation of these results requires global simulations.

- In agreement with the expectations resulting from 2D numerical simulations of viscous disks (Varnière and Tagger, 2006), pressure maxima are found to develop at the dead zone inner edge (Dzyurkevich et al., 2010). Using similar simulations, Lyra and Mac Low (2012) found that vortices grow at that location as a result of the Rossby wave instability (Lovelace et al., 1999). Such vortices are efficient trapping structures of dust particles (Barge and Sommeria, 1995) and could thus be ideal locations to initiate planet formation.

It should be noted, though, that the published simulations lack two key physical ingredients that are potentially important. One is the absence of a mean vertical field, which is unfortunate as this is the most natural magnetic field configuration. The second is that magnetic diffusivity is treated as being due to ohmic diffusion only. Ambipolar diffusion and the Hall effect, which we have seen are important in the disk upper layers, are simply ignored. Including both ingredients is only starting and we briefly mention the exciting early results that have been obtained in the next section.

Disk with vertical magnetic fields: linking disk and jets/winds As discussed in section 3.1, the most natural configuration of a disk threaded by a nonzero vertical magnetic field was long put aside due to early numerical difficulties. In addition to being important for our overall understanding of angular momentum transport in disk, such configurations are also known to lead to jets/winds launching by the disks (see the chapter by J.Ferreira for more details). Such models generally require two ingredients: a near equipartition magnetic field $(\beta \sim 1)$ as well as efficient magnetic field radial diffusion. The latter is required to prevent the magnetic field lines from accumulating near the central star. It is believed to result from disk turbulence. The problem is that the MRI is a weak field instability that is stabilized when the field reaches equipartition (see section 22). Thus the dynamical state of the disk in that regime is subject to large uncertainty. Recently, a set of papers returned to that particular geometry using stratified shearing boxes 

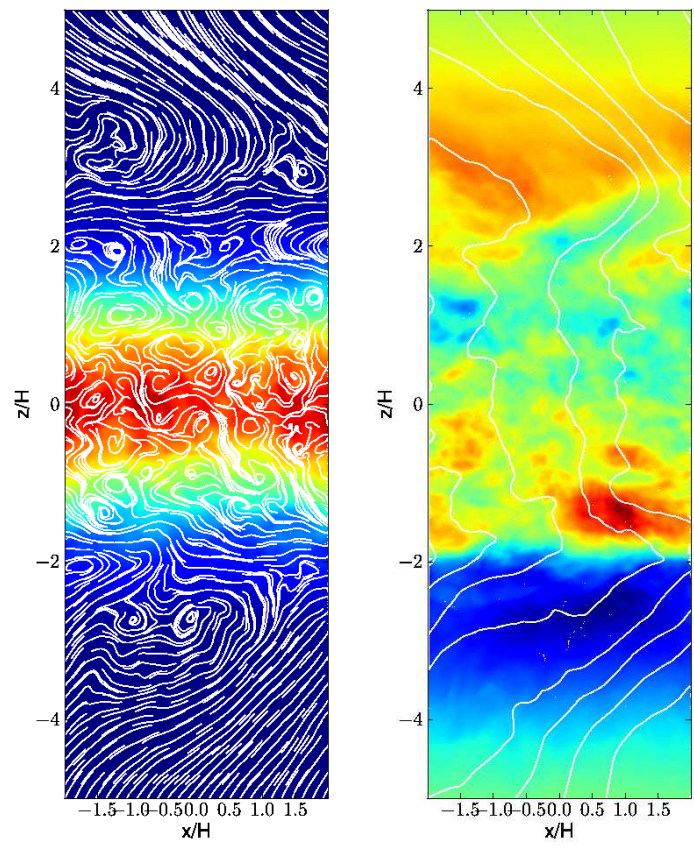

Fig. 12. Structure of the horizontally averaged poloidal streamlines (left panel) and poloidal magnetic field lines (right panel) obtained in a stratified shearing box simulation in the presence of a net vertical field (such that the midplane $\beta=10^{4}$ ) overplotted on the density distribution (left panel) and azimuthal magnetic field distribution (right panel). From Fromang et al. (2013).

simulations (still with a simplified isothermal equation of state). Several results were obtained:

- The difficulties encountered by Miller and Stone (2000) can be solved by letting the flow develop a quasi steady state in the absence of vertical field, before gradually adding that field over a few dynamical timescales (Bai and Stone, 2013a). This procedure prevents the MRI channel flows from growing and destroying the disk. Vigorous turbulence ensues when the disk reaches a quasi steady state, with $\alpha$ values up to 1 when the midplane magnetic field strength is such that $\beta=10^{2}$.

- Regardless of the field strength, the disk launches a powerful outflow out of the computational box (Suzuki and Inutsuka, 2009; Suzuki et al., 2010; Moll, 2012; Bai and Stone, 2013a; Fromang et al., 2013; Lesur et al., 2013), the properties of which resembles the classical disk wind solution first described by Blandford and Pavne (1982). The time averaged flow structure obtained in a typical simulation of that kind is illustrated on figure 12. The mass loss rate is 
significant but has been found to be sensitive to numerical details of the simulations such as box size. Whether the outflow is successful or fall back on the disk also remains to be established. Both aspects might be related to limits of the shearing box model and will probably require global simulations to be solved. Needless to say, such simulations represent a formidable task at the limit of present day supercomputers.

These recent results represent significant improvements in our ability to design realistic numerical simulations of turbulent accretion disks. They are not good representations, however, of the planet forming region in PP disks. As discussed extensively above, non-ideal MHD effects are dynamically important: ohmic diffusion dominates in the disk midplane while the Hall effect and ambipolar diffusion are important in the disk upper layers. Recently, Bai and Stone (2013b) considered such a situation by performing simulations of stratified shearing boxes with a weak net vertical field ( $\beta=10^{5}$ in the disk midplane) including ohmic and ambipolar term in the induction equation. The vertical profile of both terms were interpolated from a pre-computed lookup table which was calculated from evolving a large chemical network to equilibrium. They find that ambipolar diffusion makes qualitative changes to the structure of the flow: the otherwise turbulent surface layers of PP disks is stabilized by the ambipolar term and an outflow that takes away mass and angular momentum is launched from these laminar layers. The disk evolves toward a steady state with efficient outflow mediated angular momentum transport. This is a major evolution compared to the standard dead zone paradigm pictured in figure 4 Its robustness should be carefully assessed by future work. This will certainly require combining shearing boxes and global numerical simulations and represents a major challenge for future years.

Other transport mechanisms So far, we have concentrated on the MRI and its consequences as the only route to extract angular momentum from PP disks. What about pure hydrodynamical processes? Such processes, if they exist, could be dominant in the dead zone where the MRI does not operate. In other disk regions, they will coexist with MRI-induced turbulence and we may ask if such an interaction has important dynamical consequences.

The first question to address is that of the existence of such hydrodynamical processes. As noted in the introduction and in section 2, accretion disks are linearly stable according to the Rayleigh criterion. However, because of the enormous value of the Reynolds number, they might be nonlinearly unstable (as simple Couette flow in the laboratory). This has been the subject of intense controversy in the last twenty years. On the theoretical side, the first results have suggested that the flow remains laminar (Hawley et al., 1999) because of the stabilizing effect of the Coriolis force. This has recently been confirmed both numerically (Lesur and Longaretti, 2005) as well as using semi-analytical techniques (Rincon et al., 2007). Experiments of Taylor-Couette flows confined between two rotating cylinders have also recently been designed to study that problem. They have yielded conflicting results: Ji et al. (2006) and Schartman et al. (2012) find the flow remains laminar up to Reynolds numbers of order a million 
while Paoletti and Lathrop (2011) report signatures of a turbulent flow for similar Reynolds numbers. The interpretation of these experiments, however, is complex because of the presence of axial boundaries that produce Ekman layers (Avila, 2012). The nature of the flow in these Taylor-Couette experiment is thus unclear. Progress should be made on this issue before applying the results to PP disks.

When the equation of state of the flow is not barotropic, vorticity can be produced in the flow. This opens up the possibility for the growth of the baroclinic instability (Petersen et al., 2007; Lesur and Papaloizou, 2010). Detailed studies of the saturation of the baroclinic instability are required, but the first results (Raettig et al., 2013) suggest $\alpha$ values of order a few times $10^{-3}$. In any case, the dynamical influence of the baroclinic instability will be confined to the dead zone, as it is completely overwhelmed by MRI-induced MHD turbulence in the regions where the latter operates (Lyra and Klahr, 2011).

When their mass becomes large, PP disks start to be influenced by their own gravitational field. The importance of self-gravity is quantified by a dimensionless number called the Toomre parameter:

$$
Q=\frac{c_{0} \kappa}{\pi G \Sigma} .
$$

The rule of thumb is that self-gravity is negligible when $Q \gg 1$. When it approaches unity, gravitational instabilities develop. They take the form of spiral arms as seen in galaxies. In order to get a feel for when a given disk enters such a regime, it is enlightening to express $Q$ in terms of both the central and the disk masses. Using the explicit radius dependency for $\Omega$ and the relation $H=c_{0} / \Omega$, we can write

$$
Q \sim\left(\frac{H}{R}\right)\left(\frac{M}{M_{d}}\right),
$$

where $M_{d}=\pi R^{2} \Sigma$ is the approximate disk mass contained in the disk within a radius $R$. For PP disks where $H / R \sim 0.1$, the above expression states that selfgravity effects become important when the disk mass becomes of order one tenth of the central mass or larger. This is in general larger than the typical values of PP disks masses, although the latter are still uncertain and subject to controversy (Bergin et al., 2013). At the limit when $Q$ reaches one or smaller values, the disk is expected to become strongly unstable to non-axisymmetric modes and fragment. This has led to speculations that planets might form as a result of gravitational instabilities. The discussion of whether this actually happens or not in real systems is much beyond the scope of this lecture and could in fact be a lecture by itself. The interested reader might want to consult the short review of that problem by Stamatellos (2013) and references therein. For the purpose of this course, we just note that the issue of planet formation is far from being settled, as shown by two recent papers (Paardekooper, 2012; Meru and Bate, 2012) that illustrate some of the numerical difficulties that still remains despite years of investigations.

This discussion on the possible links between planet formation and gravitational instability naturally leads us to the examine the possible effect the MRI might have on planet formation processes. This is the purpose of the following section. 


\subsection{Planet formation}

According to the recent results of the Kepler mission (Batalha et al., 2013), exoplanets are ubiquitous in the universe. While the exact formation mode is still debated, there is a widespread consensus that planets are born in PP disks before gas dissipates. The two competing (and maybe not mutually excluding) scenarii of planet formation rely on gravitational instability (see section 4.1 above) and on the so-called core accretion model (Pollack et al., 1996). In the latter scenario planets gradually increase in mass by accumulation of solids, forming planetesimals, earth mass planets, and, when reaching a large enough mass, accreting an envelope to form gas giant planets like Jupiter. The purpose of the present section is to illustrate the typical consequences of the MRI on that process through two examples: dust dynamics (and more specifically, dust settling toward the disk equatorial plane) and planet disk interaction.

There are many more aspects of the problem that are not covered here. Particularly important are the formation of planetesimals (Johansen et al., 2007) and their subsequent evolution (see for example Gressel et al., 2012, and references therein), for which the influence of MHD turbulence is important. The MRI has also been considered in the context of on many other aspects of planet formation, such as collisions between dust particles (Carballido et al., 2010) or chondrules formation (McNally et al., 2013) to name just a few examples.

Dust dynamics in turbulent PP disks Dust dynamics is dominated by three effects: vertical settling toward the disk midplane, radial migration toward the central star and growth through coagulation. Here we focus on the first two processes, which are due to the drag force between gas and solid particles. For illustrative purposes, we consider the case of small dust particles only. When their size is smaller than the molecules mean free path (see section 1), we are in the so-called Epstein regime, in which the particles feel a drag force $\boldsymbol{F}_{\boldsymbol{d}}$ from the gas that takes the simple form

$$
\boldsymbol{F}_{\boldsymbol{d}}=-\frac{\boldsymbol{v}-\boldsymbol{v}_{\boldsymbol{d}}}{\tau_{s}}, \text { where } \tau_{s}=\frac{\rho_{s} a}{\rho c_{s}} .
$$

In the above expression, $\boldsymbol{v}_{\boldsymbol{d}}$ stands for the dust velocity and $\tau_{s}$ is the dust stopping time. This is the typical time it takes for dust particles initially at rest to reach the local gas velocity. It depends on the dust particle internal mass density $\rho_{s}$ and their size $a . \tau_{s}$ can be compared with the local dynamical time by defining the Stokes number $S t=\Omega \tau_{s}$. When $S t \ll 1$, the stopping time is much smaller than the orbital period $T_{\text {orb }}$ and the dust essentially follows the gas. When $S t \sim 1$ or larger, $\tau_{s}$ becomes comparable to $T_{\text {orb }}$ and dust and gas start to decouple.

The consequences of that friction force are simple to describe. Let's consider a single particle initially at rest (in a rotating frame) at a given radius $R_{d}$ and height $Z_{d}$ above the midplane. In the absence of gas, this particle rotates around the central star on inclined orbits at the Keplerian frequency. In a frame rotating at that frequency, the particle oscillates around the disk midplane. In the presence of gas 

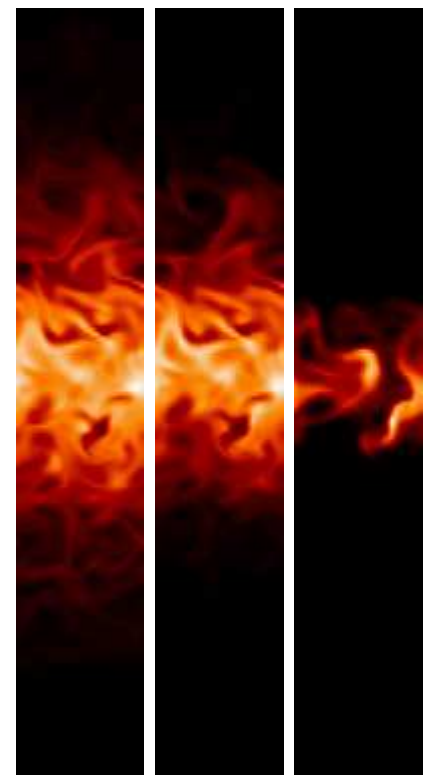

Fig. 13. Dust distribution in the $(R, Z)$ plane in a stratified shearing box numerical simulations. From left to right, the different panels correspond to $S t=0.001,0.01$ and 0.1. From Fromang and Papaloizou (2006)

in hydrostatic equilibrium, two effects appear: the moving particle experience a head wind due to the gas as a result of this oscillation. This force damps the particle oscillation. As a result, the particle falls toward the disk midplane, a process known as gravitational settling. A second effect arises because gas rotates at subKeplerian frequencies around the central object (this is due to partial support due to the negative radial pressure gradient). Thus dust particles rotate faster than the gas, experience a head wind and lose angular momentum, migrating inward in the disk.

Turbulence affects the above picture by randomly diffusing particles. In the case of gravitational settling, it prevents dust particles from collapsing into an infinitely thin layer. The effect of turbulence can be modeled as a diffusive process. If $D$ stands for a turbulent diffusion coefficient, the vertical evolution of the dust density can be described by the following partial differential equation (Dubrulle et al., 1995; Schräpler and Henning, 2004; Dullemond and Dominik, 2004):

$$
\frac{\partial \rho_{d}}{\partial t}-\frac{\partial}{\partial z}\left(z \Omega^{2} \tau_{s} \rho_{d}\right)=\frac{\partial}{\partial z}\left[D \rho \frac{\partial}{\partial z}\left(\frac{\rho_{d}}{\rho}\right)\right]
$$

where $\rho_{d}$ is the dust particle density. This equation models the balance between vertical settling and turbulent diffusion. In steady state, assuming that the dif- 
fusion coefficient $D$ is constant and the gas density vertical profile is a Gaussian, Eq. (4.4) can be integrated analytically to give

$$
\rho_{d}=\rho_{d}^{0} \exp \left[-S\left(\exp \left(\frac{Z^{2}}{2 H^{2}}\right)-1\right)-\frac{Z^{2}}{2 H^{2}}\right] \propto \exp \left[-(1+S) \frac{Z^{2}}{2 H^{2}}\right]
$$

where $\rho_{d}^{0}$ is the midplane dust density. The parameter $S=S t^{0} /\left(D / c_{s} H\right)$ measures the relative importance of the turbulent diffusion and the midplane Stokes number (Jacquet et al., 2012). The last part of Eq. (4.5) is obtained as a result of an expansion in the strong settling limit $Z \ll H$. Eq. (4.5) quantifies the effect of turbulence on gravitational settling: when $S \gg 1$, the dust layer has a thickness $H_{d}=H / \sqrt{1+S}$, i.e. it is much smaller than that of the gas (one should be careful, though, as the fluid approximation used to establish eq. (4.4) breaks down in the large Stokes number limit, see e.g. Garaud et al., 2004; Youdin and Lithwick, 2007). When $S \ll 1$, the assumption leading to the asymptotic expansion breaks down and the vertical profile for the dust is more complex but the qualitative result that $H_{d} \sim H$ still remains. The simple arguments provided here for illustrative purposes can be made more rigorous and can be extended to larger particles and to the effect of turbulence on radial dust migration (Youdin and Lithwick, 2007). In all cases, turbulence broadens the spatial distribution of a population of grains initially located at a single position in the disk.

Validation of these ideas requires detailed MHD numerical simulations in which the turbulent flow structure is self-consistently calculated. Such simulations devoted to studying vertical settling have been performed by a number of groups using local simulations in the shearing box (Johansen and Klahr, 2005; Fromang and Papaloizou, 2006; Turner et al., 2006, 2007, 2010) as well as global simulations of fully turbulent disks (Fromang and Nelson, 2009). The effect of turbulence on radial migration has been analyzed by Carballido et al. (2011). The conclusion of all these studies is the same: the dynamics induced on dust particles by the turbulent flow is well described by a diffusive process. In most instances, the simulations demonstrated quantitative agreement with analytical theories, such as that of Youdin and Lithwick (2007).

These prescriptions are now being incorporated into more realistic, but semianalytical models of PP disks that include dust coagulation (Birnstiel et al., 2012; Charnoz and Taillifet, 2012) and permit long integration times.

Planet/disk interaction in turbulent PP disks At the beginning of their lifes, young planets are still embedded in the PP disks in which they are born. Their gravitational potential adds up to that of the central star and perturb the disk structure. One manifestation of that perturbation takes the form of density waves that are excited in the disk. These waves, in turn, gravitationally torque the planet with the result of modifying their angular momentum and of changing their semi major axis: young protoplanets migrate radially (usually inward) in PP disks. The details of the interaction between the disk and the planet are complex and beyond the scope of this lecture. It has been the focus of intense research 
in the last decades and major reviews have been written in the last few years, to which the interested readers is referred (see, e.g. Baruteau and Masset, 2013, and references therein). For the purpose of this lecture, it will be enough to know that this interaction can be divided in two broad categories that depend on planet mass:

- Type I planet/disk interaction, relevant to low mass planet (typically earth to Neptune mass planets): in this case, the waves excited by the planet are linear in amplitude. They exert a torque on the planet resulting in its inward migration on timescales that can be as small as $10^{5}$ years. This fast migration represents a serious challenge for planet formation theory as the typical dissipation timescale of PP disks is longer by about an order of magnitude. Planet undergoing type I migration should then quickly reach the very vicinity of their central star, and perhaps even be swallowed by it (depending on the presence of an inner magnetospheric cavity). This problem has stimulated recent interest in studying the interaction between the planet and the gas located in its coorbital region, the so-called corotation torque. In some circumstances, it has been found that the corotation torque can reduce and even reverse type I migration.

- Type II planet/disk interaction, relevant to massive planets (with masses typically in the range of that of giant gaseous planets): in this case, the waves excited by the planet are nonlinear. Shocks form and deposit their angular momentum in the planet vicinity. Matter is evacuated from the region coorbiting with the planet, creating a gap (i.e. a low density annular region) around the planet orbit. The planet is locked inside that gap and follows the disk evolution. Planet migration occurs on viscous timescales in this case.

The structure of the disk in both situations is illustrated in figure 14. For completeness, it should be mentioned that a third regime exists, type III migration (Masset and Papaloizou, 2003). It corresponds to an intermediate mass planet in a more massive disk and can result in fast inward or outward migration. We shall not considered that case any further in the present lecture.

Most of the numerical simulations of that problem published so far have focused on planet/disk interaction in situations where the disk flow is laminar and viscous. In such simulations, viscosity is included as a large scale model of the turbulence. This approach significantly simplifies the problem. Simple situations (2D, small mass limit) are amenable to an analytical treatment that is very useful to help interpret the simulations. It also greatly reduces the computational cost associated with numerical simulations of the problem. However, it should not be forgotten that the viscous treatment of the dissipation in the disk is only a model of the effect of turbulence. As such, it has limits and can fail in some circumstances. Fortunately, thanks to the large increase in computational resources of the last few years, it is now possible to start addressing the question of the validity of that approach and to investigate the peculiarities, if any, introduced by the fact that the flow is turbulent and not laminar. The purpose of this section is to review this on-going effort.

The overall picture that emerges is as follows: in general, the results obtained using a viscous model are reasonable. They always produce results that are in 

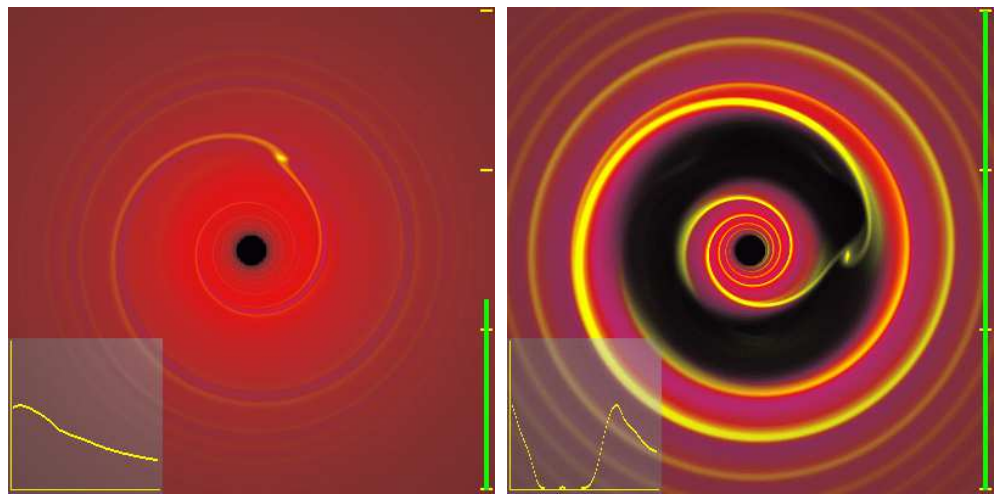

Fig. 14. Snapshots of the gas density in a PP disk when perturbed by an earth mass planet (left panel) and a Jupiter mass planet (right panel). The planet undergoes type I migration in the first case and type II migration in the second case. Inserts on the lower left hand side of each panels plot the azimutally averaged radial profile of the disk surface density. Images taken from P.Armitage website (http://jila.colorado.edu/ pja/planet_migration.html).

good qualitative agreement with the simple 2D viscous approach: for example, the disk structure as perturbed by a Jupiter mass planet in a turbulent disk is illustrated in figure 15. Aside from the turbulent density fluctuations, there is good agreement with the laminar case (right panel of figure 14). The quantitative agreement between the two types of approaches is also acceptable in most situations. For example, the migration rate of a 30 earth mass planet in a turbulent disk agrees well with the expectations based on viscous and laminar disk simulations (Nelson and Papaloizou, 2004). The gap structure is also found to be well predicted by such simple simulations (Nelson and Papaloizou, 2003; Winters et al., 2003,10

However, there are also differences some of which are important in the context of planet formation theory:

- When the planet mass is small, the amplitude of the density wave it excites is of the same order as that of the turbulent density fluctuations (see for example figure (16). This means that the torque of the later, which is random, is of comparable amplitude as that of the former. Because it is fluctuating in time, the total torque exerted by the disk on the planet is then the sum of a constant component and a fluctuating component. As a result the planet experiences a random walk in addition to its systematic inward migration. This process is now called stochastic migration (Nelson and Papaloizou, 2004). It offers a potential

\footnotetext{
${ }^{10}$ Note however that very recent results suggest that this might not be true for all magnetic field topologies (Zhu et al., 2013).
} 


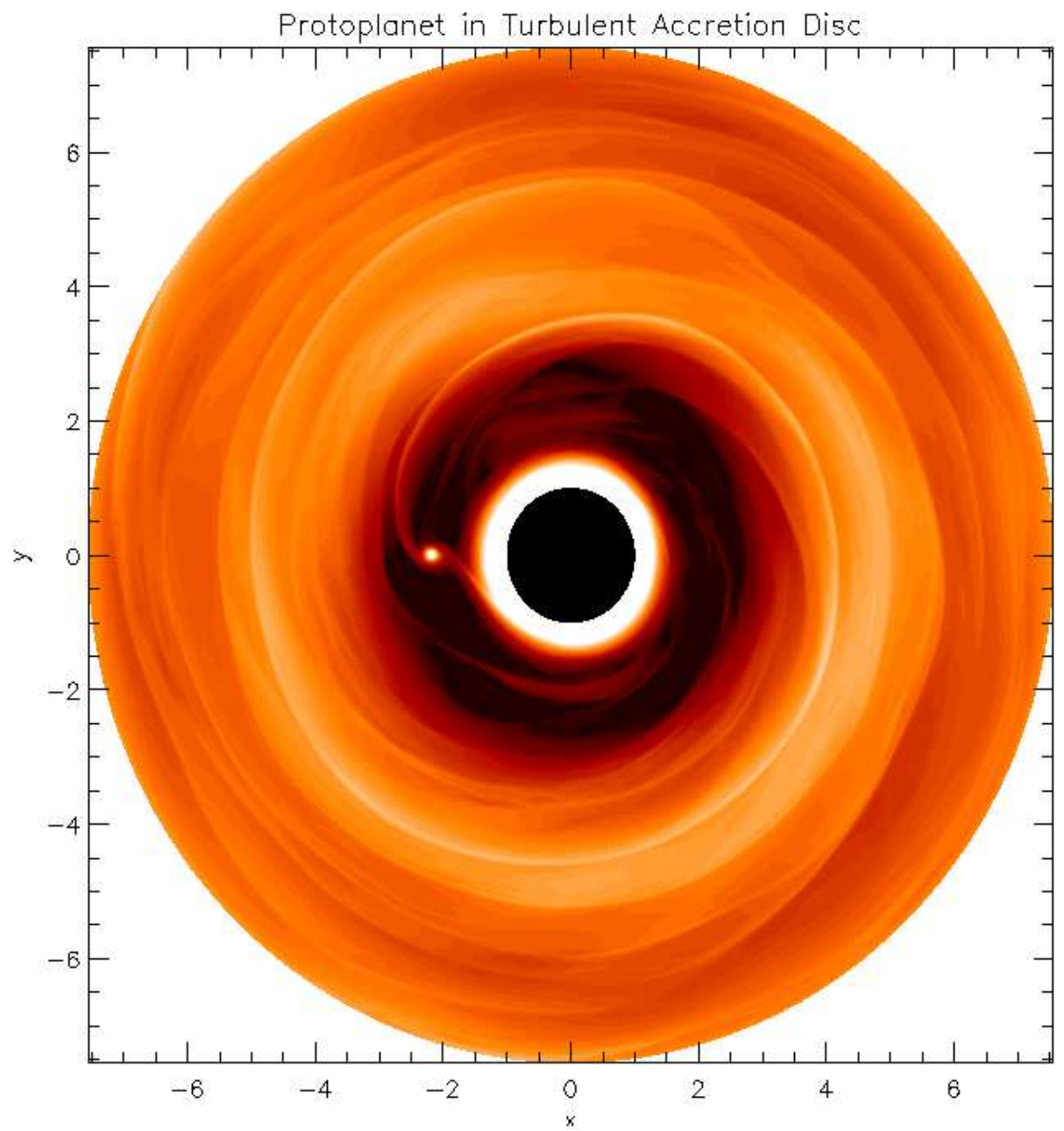

Fig. 15. Midplane gas density in a turbulent PP disks perturbed by a Jupiter mass planet. Note the presence of turbulent density fluctuations that perturbs the propagation of density waves excited by the planet. Despite the presence of the turbulence, the disk structure remains similar to that of a laminar disk such as shown on figure 14 From Nelson and Papaloizou (2003)

solution to the inward migration problem of small mass planets by reducing type I migration rates, even if the extrapolation of the simulation results to long evolutionary timescales is still debated.

- The corotation torque arises from scales comparable to the disk scaleheight, or, equivalently, comparable to the scale of the turbulence. This suggests that 

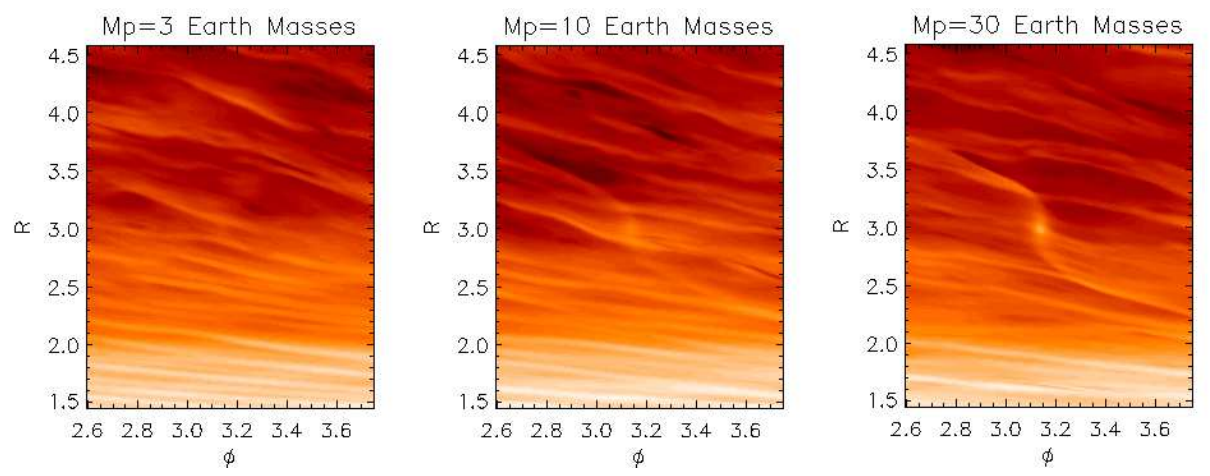

Fig. 16. Close-up view of the gas density in a PP disk perturbed by a planet of mass $M_{p}=3 \mathrm{M}_{\oplus}$ (left panel), $M_{p}=10 \mathrm{M}_{\oplus}$ (middle panel) and $M_{p}=30 \mathrm{M}_{\oplus}$ (right panel). Note the change in the relative importance of the planetary wake and of the turbulent density fluctuations as the planet mass is varied. In the first case, planet migration is completely dominated by the stochastic torque caused by these turbulent fluctuations. From Nelson and Papaloizou (2004)

a diffusive description of the effect of the turbulence is questionable. Recent global disk simulations of planet/disk interaction in turbulent disk aiming at isolating the effect of the corotation torque have been able to show that the coorbital region still creates a torque onto the planet despite the presence of the turbulence (Baruteau et al., 2011). Nevertheless, the properties of that torque (i.e. its scaling with the disk physical parameters and with the magnetic field) display departure from the predictions of a pure viscous model that should be investigated in future simulations systematically covering a wider range of conditions.

It should be emphasize that published studies of the effect of MHD turbulence on planet/disk interaction have so far been limited to idealized situations. A major challenge of future years will be to investigate the modifications of this picture that result from the complex structure of PP disks highlighted in previous sections.

\section{Conclusions}

At the time of concluding this lecture, it is important to emphasize once more that it has no ambition but being a concise (and thus, incomplete) introduction to the question of MRI-driven angular momentum transport. Several aspects of the problem are only partially covered or even completely ignored. This is because the field is so vast. The study of angular momentum transport in PP disks is interdisciplinary in nature. It brings together various aspects of modern astrophysics such as plasma physics, fluid dynamics, chemistry and radiative transfer and intimately mixes analytical and numerical approaches. My hope is that the variety of topics 
introduced in this lecture will stimulate the interest of the reader. The references that are given along the way are as many starting points for further readings.

Before closing, and maybe to stimulate discussions, controversy, future research and hopefully progress, this lecture should end with an humble note. Despite the impressive achievements of the past twenty years, it is fair to say that we still don't know with enough confidence at which rate angular momentum is being transported in PP disks. Their structure is still highly uncertain. As highlighted by the last part of this lecture, this is not without consequences for several aspects of planet formation. Much remains to be done before we can form a self-consistent picture of how planets form in the universe.

\section{Acknowledgment}

I am indebted to all the colleagues that contributed to my understanding of the field over the past ten years. This lecture would never have come to be without their support and communicative interest for the subjects of accretion disk dynamics and planet formation. I also acknowledge Xuening Bai, Steven Balbus, Clement Baruteau, Arnaud Belloche and Geoffroy Lesur for a careful reading of an earlier draft of this lecture.

\section{References}

Armitage, P. J.: 1998, ApJ 501, L189

Armitage, P. J.: 2011, ARAA 49, 195

Avila, M.: 2012, Physical Review Letters 108(12), 124501

Bai, X.-N.: 2011a, ApJ 739, 50

Bai, X.-N.: 2011b, ApJ 739, 51

Bai, X.-N. and Stone, J. M.: 2011, ApJ 736, 144

Bai, X.-N. and Stone, J. M.: 2013a, ApJ 767, 30

Bai, X.-N. and Stone, J. M.: 2013b, ArXiv e-prints

Balbus, S. and Hawley, J.: 1991, ApJ 376, 214

Balbus, S. and Hawley, J.: 1998, Rev.Mod.Phys. 70, 1

Balbus, S. A.: 2003, ARAA 41, 555

Balbus, S. A.: 2011, Magnetohydrodynamics of Protostellar Disks, pp 237-282

Balbus, S. A. and Hawley, J. F.: 1992, ApJ 392, 662

Balbus, S. A. and Hawley, J. F.: 2000, in From Dust to Terrestrial Planets, p. 39

Balbus, S. A. and Terquem, C.: 2001, ApJ 552, 235 
46Angular momentum transport during the formation and early evolution of stars - EES2012

Barge, P. and Sommeria, J.: 1995, A\&A 295, L1

Baruteau, C., Fromang, S., Nelson, R. P., and Masset, F.: 2011, Aש̈A 533, A84

Baruteau, C. and Masset, F.: 2013, in J. Souchay, S. Mathis, and T. Tokieda (eds.), Lecture Notes in Physics, Berlin Springer Verlag, Vol. 861 of Lecture Notes in Physics, Berlin Springer Verlag, p. 201

Batalha, N. M., Rowe, J. F., Bryson, S. T., Barclay, T., Burke, C. J., Caldwell, D. A., Christiansen, J. L., Mullally, F., Thompson, S. E., Brown, T. M., Dupree, A. K., Fabrycky, D. C., Ford, E. B., Fortney, J. J., Gilliland, R. L., Isaacson, H., Latham, D. W., Marcy, G. W., Quinn, S. N., Ragozzine, D., Shporer, A., Borucki, W. J., Ciardi, D. R., Gautier, III, T. N., Haas, M. R., Jenkins, J. M., Koch, D. G., Lissauer, J. J., Rapin, W., Basri, G. S., Boss, A. P., Buchhave, L. A., Carter, J. A., Charbonneau, D., Christensen-Dalsgaard, J., Clarke, B. D., Cochran, W. D., Demory, B.-O., Desert, J.-M., Devore, E., Doyle, L. R., Esquerdo, G. A., Everett, M., Fressin, F., Geary, J. C., Girouard, F. R., Gould, A., Hall, J. R., Holman, M. J., Howard, A. W., Howell, S. B., Ibrahim, K. A., Kinemuchi, K., Kjeldsen, H., Klaus, T. C., Li, J., Lucas, P. W., Meibom, S., Morris, R. L., Prša, A., Quintana, E., Sanderfer, D. T., Sasselov, D., Seader, S. E., Smith, J. C., Steffen, J. H., Still, M., Stumpe, M. C., Tarter, J. C., Tenenbaum, P., Torres, G., Twicken, J. D., Uddin, K., Van Cleve, J., Walkowicz, L., and Welsh, W. F.: 2013, ApJS 204, 24

Beckwith, K., Armitage, P. J., and Simon, J. B.: 2011, MNRAS 416, 361

Bergin, E. A., Cleeves, L. I., Gorti, U., Zhang, K., Blake, G. A., Green, J. D., Andrews, S. M., Evans, II, N. J., Henning, T., Öberg, K., Pontoppidan, K., Qi, C., Salyk, C., and van Dishoeck, E. F.: 2013, Nature 493, 644

Birnstiel, T., Klahr, H., and Ercolano, B.: 2012, A\&GA 539, A148

Blaes, O. M. and Balbus, S. A.: 1994, ApJ 421, 163

Blandford, R. D. and Payne, D. G.: 1982, MNRAS 199, 883

Bodo, G., Cattaneo, F., Ferrari, A., Mignone, A., and Rossi, P.: 2011, ApJ 739, 82

Brandenburg, A., Nordlund, A., Stein, R. F., and Torkelsson, U.: 1995, ApJ 446, 741

Carballido, A., Bai, X.-N., and Cuzzi, J. N.: 2011, MNRAS 415, 93

Carballido, A., Cuzzi, J. N., and Hogan, R. C.: 2010, MNRAS 405, 2339

Chandrasekhar, S.: 1961, Hydrodynamic and hydromagnetic stability

Charnoz, S. and Taillifet, E.: 2012, ApJ 753, 119

D'Alessio, P., Canto, J., Calvet, N., and Lizano, S.: 1998, ApJ 500, 411

Davis, S. W., Stone, J. M., and Pessah, M. E.: 2010, ApJ 713, 52

Dubrulle, B., Morfill, G., and Sterzik, M.: 1995, Icarus 114, 237

Dullemond, C. P. and Dominik, C.: 2004, A\&A 421, 1075 
Dullemond, C. P. and Monnier, J. D.: 2010, ARAA 48, 205

Dzyurkevich, N., Flock, M., Turner, N. J., Klahr, H., and Henning, T.: 2010, A\&A 515, $\mathrm{A} 70+$

Fatuzzo, M., Adams, F. C., and Melia, F.: 2006, ApJL 653, L49

Flaig, M., Kley, W., and Kissmann, R.: 2010, ArXiv e-prints

Flaig, M., Ruoff, P., Kley, W., and Kissmann, R.: 2012, MNRAS 420, 2419

Fleming, T. and Stone, J. M.: 2003, ApJ 585, 908

Fleming, T. P., Stone, J. M., and Hawley, J. F.: 2000, ApJ 530, 464

Flock, M., Dzyurkevich, N., Klahr, H., Turner, N., and Henning, T.: 2012, ApJ 744, 144

Flock, M., Dzyurkevich, N., Klahr, H., Turner, N. J., and Henning, T.: 2011, ApJ 735, 122

Frank, J., King, A., and Raine, D. J.: 2002, Accretion Power in Astrophysics: Third Edition

Fromang, S., Hennebelle, P., and Teyssier, R.: 2006, A\&\&A 457, 371

Fromang, S., Latter, H., Lesur, G., and Ogilvie, G. I.: 2013, A\&A 552, A71

Fromang, S., Lyra, W., and Masset, F.: 2011, A\&\&A 534, A107

Fromang, S. and Nelson, R. P.: 2006, A\&A 457, 343

Fromang, S. and Nelson, R. P.: 2009, A\&A 496, 597

Fromang, S. and Papaloizou, J.: 2006, AESA 452, 751

Fromang, S. and Papaloizou, J.: 2007, A\&A 476, 1113

Fromang, S., Papaloizou, J., Lesur, G., and Heinemann, T.: 2007, A\&A 476, 1123

Fromang, S., Terquem, C., and Balbus, S. A.: 2002, MNRAS 329, 18

Gammie, C. F.: 1996, ApJ 457, 355

Gammie, C. F. and Balbus, S. A.: 1994, MNRAS 270, 138

Garaud, P., Barrière-Fouchet, L., and Lin, D. N. C.: 2004, ApJ 603, 292

Glassgold, A. E., Najita, J., and Igea, J.: 1997, ApJ 480, 344

Goodman, J. and Xu, G.: 1994, ApJ 432, 213

Gressel, O.: 2010, MNRAS 405, 41

Gressel, O., Nelson, R. P., and Turner, N. J.: 2012, MNRAS 422, 1140

Guan, X., Gammie, C. F., Simon, J. B., and Johnson, B. M.: 2009, ApJ 694, 1010 
48Angular momentum transport during the formation and early evolution of stars - EES2012

Hawley, J. and Stone, J.: 1995, Comput. Phys. Commun. 89, 127

Hawley, J. F.: 2001, ApJ 554, 534

Hawley, J. F. and Balbus, S. A.: 1991, ApJ 376, 223

Hawley, J. F. and Balbus, S. A.: 1992, ApJ 400, 595

Hawley, J. F., Balbus, S. A., and Winters, W. F.: 1999, ApJ 518, 394

Hawley, J. F., Gammie, C. F., and Balbus, S. A.: 1995, ApJ 440, 742

Hawley, J. F., Gammie, C. F., and Balbus, S. A.: 1996, ApJ 464, 690

Hawley, J. F. and Stone, J. M.: 1998, ApJ 501, 758

Herault, J., Rincon, F., Cossu, C., Lesur, G., Ogilvie, G. I., and Longaretti, P.-Y.: 2011, PhysRevE 84(3), 036321

Hirose, S. and Turner, N. J.: 2011, ApJL 732, L30

Hueso, R. and Guillot, T.: 2005, A\&\&A 442, 703

Ilgner, M. and Nelson, R. P.: 2006a, A\&A 445, 205

Ilgner, M. and Nelson, R. P.: 2006b, A\& A 445, 223

Iskakov, A., Schekochihin, A., Cowley, S., McWilliams, J. C., and Proctor, M. R. E.: 2007, Physical Review Letters 98, 208501

Jacquet, E., Gounelle, M., and Fromang, S.: 2012, Icarus 220, 162

Ji, H., Burin, M., Schartman, E., and Goodman, J.: 2006, Nature 444, 343

Johansen, A. and Klahr, H.: 2005, ApJ 634, 1353

Johansen, A., Oishi, J. S., Low, M., Klahr, H., Henning, T., and Youdin, A.: 2007, Nature 448, 1022

Latter, H. N. and Balbus, S.: 2012, MNRAS 424, 1977

Lesur, G., Ferreira, J., and Ogilvie, G. I.: 2013, A\& A 550, A61

Lesur, G. and Longaretti, P.-Y.: 2005, A\&A 444, 25

Lesur, G. and Longaretti, P.-Y.: 2007, MNRAS 378, 1471

Lesur, G. and Papaloizou, J. C. B.: 2010, A\&A 513, A60

Longaretti, P. and Lesur, G.: 2010, A\&A submitted

Lovelace, R. V. E., Li, H., Colgate, S. A., and Nelson, A. F.: 1999, ApJ 513, 805

Lynden-Bell, D. and Pringle, J. E.: 1974, MNRAS 168, 603

Lyra, W. and Klahr, H.: 2011, A\&SA 527, A138 
Lyra, W. and Mac Low, M.-M.: 2012, ApJ 756, 62

Mac Low, M. and Klessen, R. S.: 2004, Reviews of Modern Physics 76, 125

Martin, R. G., Lubow, S. H., Livio, M., and Pringle, J. E.: 2012, MNRAS 423, 2718

Masset, F. S. and Papaloizou, J. C. B.: 2003, ApJ 588, 494

McNally, C. P., Hubbard, A., Mac Low, M.-M., Ebel, D. S., and D'Alessio, P.: 2013, ArXiv e-prints

Meru, F. and Bate, M. R.: 2012, MNRAS 427, 2022

Mignone, A., Bodo, G., Massaglia, S., Matsakos, T., Tesileanu, O., Zanni, C., and Ferrari, A.: 2007, ApJS 170, 228

Miller, K. A. and Stone, J. M.: 2000, ApJ 534, 398

Moll, R.: 2012, ArXiv e-prints

Nelson, R. and Papaloizou, J.: 2003, MNRAS 339, 993

Nelson, R. P. and Papaloizou, J. C. B.: 2004, MNRAS 350, 849

Oppenheimer, M. and Dalgarno, A.: 1974, ApJ 192, 29

Paardekooper, S.-J.: 2012, MNRAS 421, 3286

Paoletti, M. S. and Lathrop, D. P.: 2011, Physical Review Letters 106(2), 024501

Papaloizou, J. C. B. and Nelson, R. P.: 2003, MNRAS 339, 983

Perez-Becker, D. and Chiang, E.: 2011, ApJ 735, 8

Pessah, M. E. and Chan, C.-k.: 2008, ApJ 684, 498

Pessah, M. E., Chan, C.-k., and Psaltis, D.: 2007, ApJL 668, L51

Petersen, M. R., Julien, K., and Stewart, G. R.: 2007, ApJ 658, 1236

Pollack, J., Hubickyj, O., Bodenheimer, P., Lissauer, J., Podolak, M., and Greenzweig, Y.: 1996, Icar 124, 62

Raettig, N., Lyra, W., and Klahr, H.: 2013, ApJ 765, 115

Rincon, F., Ogilvie, G. I., and Cossu, C.: 2007, A\&A 463, 817

Sano, T. and Stone, J. M.: 2002a, ApJ 570, 314

Sano, T. and Stone, J. M.: 2002b, ApJ 577, 534

Schartman, E., Ji, H., Burin, M. J., and Goodman, J.: 2012, A 6 A 543, A94

Schekochihin, A. A., Cowley, S. C., Taylor, S. F., Maron, J. L., and McWilliams, J. C.: 2004, ApJ 612, 276

Schräpler, R. and Henning, T.: 2004, ApJ 614, 960 
50Angular momentum transport during the formation and early evolution of stars - EES2012

Shakura, N. I. and Sunyaev, R. A.: 1973, A\& A 24, 337

Shi, J., Krolik, J. H., and Hirose, S.: 2010, ApJ 708, 1716

Simon, J. B., Bai, X.-N., Stone, J. M., Armitage, P. J., and Beckwith, K.: 2013, ApJ $\mathbf{7 6 4}, 66$

Simon, J. B., Beckwith, K., and Armitage, P. J.: 2012, MNRAS 422, 2685

Simon, J. B. and Hawley, J. F.: 2009, ApJ 707, 833

Simon, J. B., Hawley, J. F., and Beckwith, K.: 2009, ApJ 690, 974

Simon, J. B., Hawley, J. F., and Beckwith, K.: 2011, ApJ 730, 94

Sorathia, K. A., Reynolds, C. S., and Armitage, P. J.: 2010, ApJ 712, 1241

Sorathia, K. A., Reynolds, C. S., Stone, J. M., and Beckwith, K.: 2012, ApJ 749, 189

Spitzer, L.: 1962, Physics of Fully Ionized Gases

Stamatellos, D.: 2013, ArXiv e-prints

Steinacker, A. and Papaloizou, J.: 2002, ApJ 571, 413

Stone, J. M., Gardiner, T. A., Teuben, P., Hawley, J. F., and Simon, J. B.: 2008, ApJS 178, 137

Stone, J. M., Hawley, J. F., Gammie, C. F., and Balbus, S. A.: 1996, ApJ 463, 656

Stone, J. M. and Norman, M. L.: 1992a, ApJS 80, 753

Stone, J. M. and Norman, M. L.: 1992b, ApJS 80, 791

Suzuki, T. K. and Inutsuka, S.-i.: 2009, ApJL 691, L49

Suzuki, T. K., Muto, T., and Inutsuka, S.-i.: 2010, ApJ 718, 1289

Terquem, C. E. J. M. L. J.: 2008, ApJ 689, 532

Teyssier, R.: 2002, A\&A 385, 337

Toro, E.: 1997, Riemann solvers and numerical methods for fluid dynamics, Springer

Turner, N. J., Carballido, A., and Sano, T.: 2010, ApJ 708, 188

Turner, N. J. and Drake, J. F.: 2009, ApJ 703, 2152

Turner, N. J., Sano, T., and Dziourkevitch, N.: 2007, ApJ 659, 729

Turner, N. J., Willacy, K., Bryden, G., and Yorke, H. W.: 2006, ApJ 639, 1218

Umebayashi, T. and Nakano, T.: 1988, Progress of Theoretical Physics Supplement 96, 151

Umurhan, O. M. and Regev, O.: 2004, A\&A 427, 855 
Varnière, P. and Tagger, M.: 2006, A\&A 446, L13

Velikhov, E. P.: 1959, Sov. Phys. JETP 36, 995

Wardle, M.: 1997, in D. T. Wickramasinghe, G. V. Bicknell, and L. Ferrario (eds.), IAU Colloq. 163: Accretion Phenomena and Related Outflows, Vol. 121 of Astronomical Society of the Pacific Conference Series, p. 561

Wardle, M.: 1999, MNRAS 307, 849

Wardle, M. and Salmeron, R.: 2012, MNRAS 422, 2737

Winters, W. F., Balbus, S. A., and Hawley, J. F.: 2003, ApJ 589, 543

Youdin, A. N. and Lithwick, Y.: 2007, Icarus 192, 588

Zhu, Z., Hartmann, L., and Gammie, C.: 2010a, ApJ 713, 1143

Zhu, Z., Hartmann, L., Gammie, C. F., Book, L. G., Simon, J. B., and Engelhard, E.: 2010b, ApJ 713, 1134

Zhu, Z., Stone, J. M., and Rafikov, R. R.: 2013, ArXiv e-prints

Ziegler, U.: 2004, Journal of Computational Physics 196, 393

Ziegler, U.: 2008, Computer Physics Communications 179, 227 